\title{
34.5. THE MEDITERRANEAN DIATOMS
}

\author{
Marta Hajós, Hungarian Geological Institute, Budapest, Hungary
}

\section{INTRODUCTION}

Ten washed samples taken at eight levels from the drill cores of the Mediterranean expedition of the Deep Sea Drilling Project were sent for study to the present writer by Paulian Dumitrica of the Geological Institute, Bucharest, Rumania. He had found these samples to be the richest diatom-bearing sediments both in terms of species and individuals.

Two samples from Core 13 of Site 124-Balearic Rise ${ }^{1}$ were selected near levels of finely laminated dolomitic marls with dark green to black interbeds. This dolomitic unit is part of the evaporite series cored in the deep Balearic Basin. All six samples from Sites 127 and 128 in the Hellenic Trench of the Ionian Basin ${ }^{2}$ are from layers of dark sapropelitic ooze, where the siliceous microfauna is particularly abundant. The remaining two samples are from Site 130 on the Mediterranean Ridge in the Levantine Basin $^{3}$, and represent the siliceous microfossil content of dark blue black terrigenous clays believed to have been transported to this site from the Nile Cone (see Conclusions of Chapters 11, 25.2, and 35 of this volume).

\section{Procedures}

The author's study has been restricted to investigating the available washed samples. It must be taken into consideration that because a sizable portion of the flora may have been lost in sample preparation, the assemblages reported here may not be representative of the original thanatocoenosis, nor of the original depositional environment. The investigations were carried out on strewn slides mounted in Caedex with the aid of an Opton binocular photomicroscope; the documented taxa were photographed with the same instrument.

\section{DISCUSSION}

In the plates enclosed herewith (Plates 1-9) only a few characteristic species of the rich floral assemblages are shown. The complete floral list is shown on Table 1, with indication of the characteristic ecologies and ranges of the

\footnotetext{
${ }^{1}$ Site 124 , Latitude: $38^{\circ} 52.4^{\prime} \mathrm{N}$; Longitude: $04^{\circ} 59.7^{\prime} \mathrm{E}$; water depth 2726 meters; Core 13 at 417 meters below the sea floor.

${ }^{2}$ Site 127 , Latitude: $35^{\circ} 43.9^{\prime} \mathrm{N}$, Longitude: $22^{\circ} 29.8^{\prime} \mathrm{E}$; water depth 4654 meters; Core 5 at 101 meters and Core 14 at 380 meters below the sea floor, respectively.

Site 128 , Latitude: $35^{\circ} 42.6^{\prime} \mathrm{N}$; Longitude: $22^{\circ} 28.1^{\prime} \mathrm{E}$; water depth 4640 meters; Core 3 at 88 meters, Core 10 at 418 meters, and Core 11 at 474 meters below the sea floor, respectively.

${ }^{3}$ Site 130 , Latitude: $33^{\circ} 36.3^{\prime} \mathrm{N}$; Longitude: $27^{\circ} 52.0^{\prime} \mathrm{E}$; water depth 2979 meters; Core 3 at 86 meters and Core 4 at 150 meters below the sea floor, respectively.
}

species. The relevant information has been obtained from the references cited at the end of this chapter and from investigations of Hungarian fossiliferous localities.

The 175 taxa investigated belong primarily to the phyla Chrysophyta and Bacillariophyta, and are accompanied in subordinate numbers by representatives of Phytolitharia, Acritarcha, Radiolaria and Porifera.

The paleoecological assignments shown in Table 1 and the complete floral spectrum allow three paleo-ecological divisions, representing three areas of the Mediterranean and three different sedimentary facies, to be distinguished.

\section{Division 1 - Siliceous Dolomitic Marls of Site 124}

The sediments of the two samples from Site 124 (124-13-2, 89-90 cm and 124-13-2, 127-129 cm) were apparently deposited under the same ecological conditions. The predominantly littoral planktonic forms are accompanied by brackish water, and even freshwater, euryhaline, benthonic, and epiphytic species in considerable numbers.

Taxa characteristic of stagnant brackish waters and landlocked salinas and lagoons are present in such great numbers as to suggest reduced salinity, shallow water depth, and a landlocked, stagnant sedimentary environment. As for salinity, it may have been upper brackish ( 0 to $5 \%$ ) or possibly lower brackish $(5$ to $20 \%$ ). Since the species indicative of low salinity are benthonic, they must be mutochthonous rather than allochthonous. The littoral brackish water forms living in salinas and stagnant waters found in these samples include:

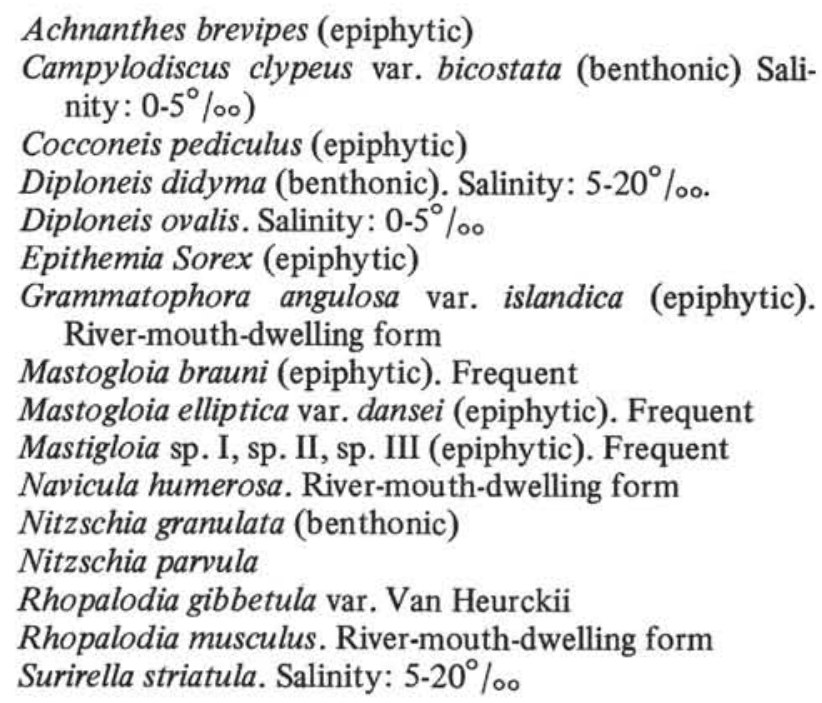
nity: $0-5 \%$ )

The presence of freshwater and river-mouth-dwelling species indicates a considerable influx of fresh waters into a landlocked basin at the time of formation of the dolomitic marls. These species include: 
TABLE 1

The Distribution of Siliceous Microfossils in the Samples Studied

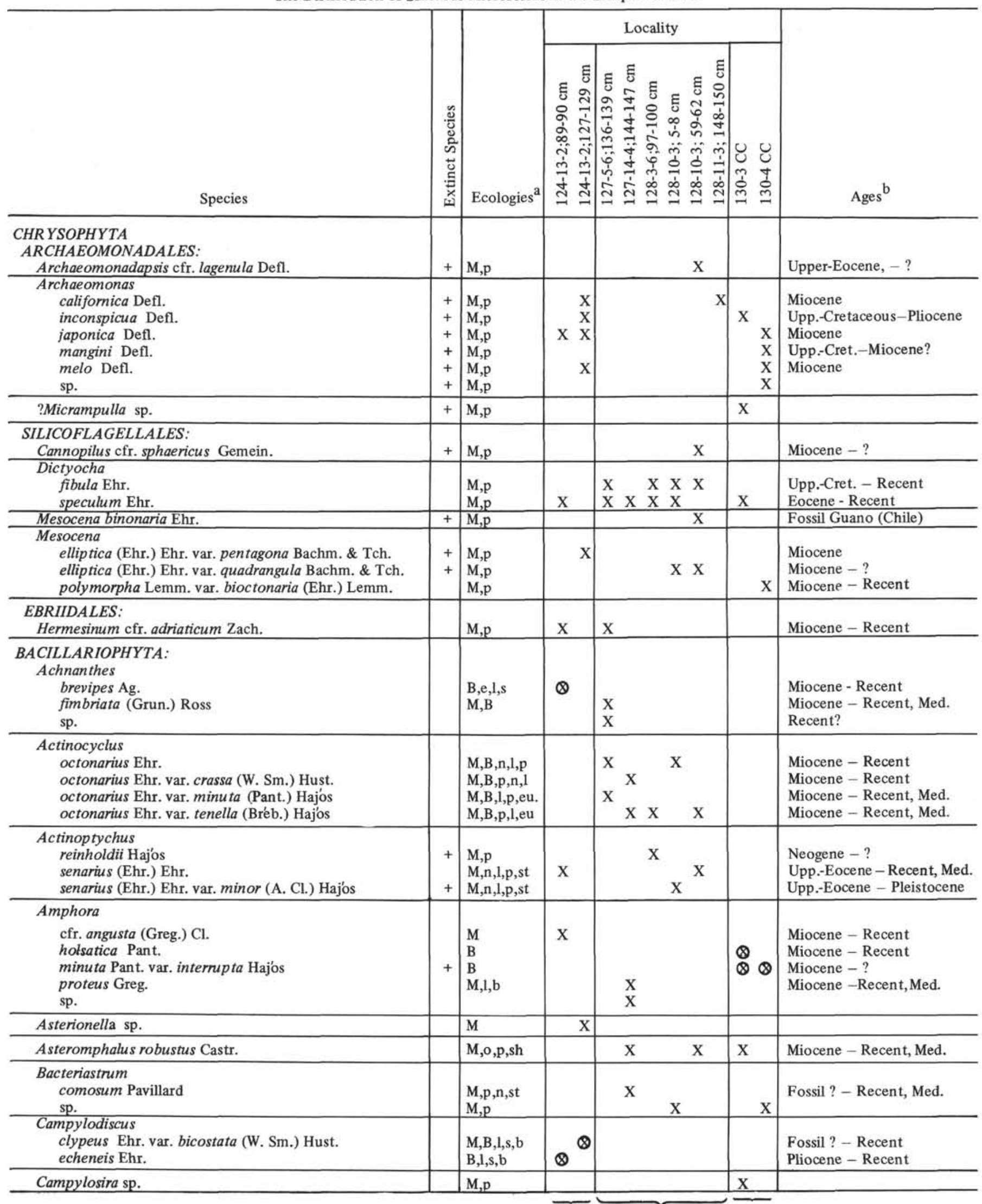


TABLE 1 - Continued

\begin{tabular}{|c|c|c|c|c|c|c|c|c|}
\hline \multirow[b]{2}{*}{ Species } & \multirow[b]{2}{*}{ 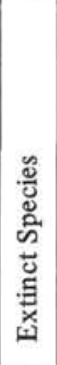 } & \multirow[b]{2}{*}{ Ecologies $^{\mathrm{a}}$} & \multicolumn{5}{|c|}{ Locality } & \multirow[b]{2}{*}{ Ages $^{b}$} \\
\hline & & & 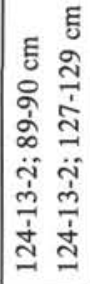 & 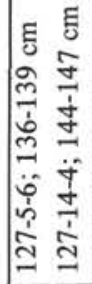 & 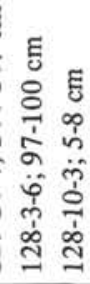 & 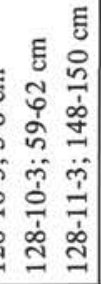 & $\mid \begin{array}{ll}0 & 0 \\
0 & 0 \\
0 & \searrow \\
0 & 0 \\
-1 & 0 \\
& -1\end{array}$ & \\
\hline $\begin{array}{l}\text { Chaetoceros } \\
\text { gastridium Ehr. } \\
\text { lauderi Ralfs } \\
\text { sp. I } \\
\text { sp. II } \\
\text { sp. III } \\
\text { sp. IV } \\
\text { sp. V } \\
\text { sp. VI } \\
\end{array}$ & & $\begin{array}{l}\text { M,p,st } \\
\text { M,p,n } \\
\mathrm{M}, \mathrm{p} \\
\mathrm{M}, \mathrm{p} \\
\mathrm{M}, \mathrm{p} \\
\mathrm{M}, \mathrm{p} \\
\mathrm{M}, \mathrm{p} \\
\mathrm{M}, \mathrm{p}\end{array}$ & $\begin{array}{l}X \\
X \\
\end{array}$ & $\mathrm{X}$ & $\begin{array}{l}X \\
X \\
X \\
X \\
X\end{array}$ & K & $\begin{array}{l}X \\
X \\
X\end{array}$ & $\begin{array}{l}\text { Fossil Guana (Peru) - Recent } \\
\text { Pleistocene - Recent }\end{array}$ \\
\hline $\begin{array}{l}\text { Cocconeis } \\
\text { britannica Naeg. } \\
\text { costata Greg. } \\
\text { ornata Greg. var. signata Perag. } \\
\text { pediculus } \text { Ehr. } \\
\text { placentula } \text { Ehr. var. klinoraphis Geitl. } \\
\text { pseudomarginata Greg. } \\
\text { scutellum } \text { Ehr. } \\
\text { sp. I } \\
\text { sp. II } \\
\end{array}$ & & $\begin{array}{l}\text { M,l,e } \\
\text { M,l,e,b } \\
\text { M,l,e,b,st. } \\
\text { B,t,e,l,s } \\
\text { F,e } \\
\text { M,l,e,b } \\
\text { M,B,e,s,b,n } \\
\text { e } \\
\text { e }\end{array}$ & $\begin{array}{l}\mathrm{X} \\
\mathrm{X}\end{array}$ & $\begin{array}{l}X \\
x\end{array}$ & & & & $\begin{array}{l}\text { Miocene - Recent, Med. } \\
\text { Miocene - Recent, Med. } \\
\text { Fossil ? Recent } \\
\text { Fossil ? Recent } \\
\text { Pliocene - Recent } \\
\text { Miocene - Recent } \\
\text { Lower Miocene - Recent }\end{array}$ \\
\hline ?Cocconeis sp. & & $\mathrm{e}$ & $\mathrm{X}$ & & & & & \\
\hline $\begin{array}{l}\text { Coscinodiscus apiculatus Ehr. var. ambigua Grun. } \\
\text { curvatulus Grun. } \\
\text { curvatulus Grun. var. arcuata Hajos } \\
\text { curvatulus Grun. var. macropunctata Hajos } \\
\text { curvatulus Grun. var. minor (Ehr.) Grun. } \\
\text { divisus Grun. } \\
\text { excentricus Ehr. } \\
\text { excentricus Ehr. var. macropora Grun. } \\
\text { globosus Hajos } \\
\text { hustedtii Jousé } \\
\text { kützingii A.Schm. } \\
\text { ? lineatus Ehr. } \\
\text { marginatus } \text { Ehr. } \\
\text { nitidus } \text { Greg. } \\
\text { oculus-iridis Ehr. } \\
\text { sp. } \\
\end{array}$ & + & $\begin{array}{l}\text { M,p } \\
\text { M,o,n,p } \\
\text { M,p } \\
\text { M,p } \\
\text { M,p } \\
\text { M,o,n,l,p } \\
\text { B,B,p,r } \\
\text { M,B,p,r } \\
\text { M,p } \\
\text { M,p } \\
\text { M,p,l } \\
\text { M,o,n,p,r } \\
\text { M,o,p,sh } \\
\text { M,o,n,l,p } \\
\text { M,o,sh,p } \\
\text { M,p }\end{array}$ & & $\begin{array}{ll}\mathrm{X} & \\
\mathrm{X} \\
\mathrm{X}\end{array}$ & $\begin{array}{l}\mathrm{X} \\
\mathrm{X} \\
\mathrm{X} \\
\mathrm{X} \\
\mathrm{X} \\
\mathrm{X}\end{array}$ & $\begin{array}{l}\mathrm{X} \\
\mathrm{X} \\
\mathrm{X} \\
\mathrm{X} \\
\mathrm{X} \\
\mathrm{X} \\
\mathrm{X} \\
\mathrm{X} \\
\mathrm{X}\end{array}$ & \begin{tabular}{l|l}
$\mathrm{X}$ & \\
$\mathrm{X}$ & \\
$\otimes$ & $\otimes$ \\
$\mathrm{X}$ & $\mathrm{X}$ \\
$\mathrm{X}$ & \\
$\mathrm{X}$ & \\
$\mathrm{X}$ & \\
\end{tabular} & $\begin{array}{l}\text { Miocene - Recent } \\
\text { Miocene - Recent } \\
\text { Miocene - ? } \\
\text { Miocene - ? } \\
\text { Miocene - Recent } \\
\text { Miocene - Recent } \\
\text { Miocene - Recent } \\
\text { Miocene - Recent } \\
\text { Miocene - ? } \\
\text { Paleocene-Miocene - ? } \\
\text { Miocene - Recent } \\
\text { Miocene - Recent } \\
\text { Eocene - Recent } \\
\text { Upp.-Eocene - Recent } \\
\text { Upp.-Eocene - Recent }\end{array}$ \\
\hline $\begin{array}{l}\text { Cyclotella } \\
\text { comta (Ehr.) Kutz } \\
\text { sp. }\end{array}$ & & $\begin{array}{l}\mathrm{F}, \mathrm{p} \\
\mathrm{F}, \mathrm{p}\end{array}$ & & & & & & Fossil ? - Recent \\
\hline Denticulata hustedtii Simonsen et Kanaya & + & $\mathrm{M}, \mathrm{p}$ & $\mathrm{X}$ & & & & & Miocene \\
\hline Diatomella balfouriana Grev. & & F,e & & & & & & Miocene - Recent \\
\hline Dimerogramma marinum (Greg.) Ralfs & & $\mathrm{M}, \mathrm{p}, \mathrm{l}$ & & & $\mathrm{X}$ & & & Miocene - Recent, Med. \\
\hline $\begin{array}{l}\text { Diploneis } \\
\text { bombus } \text { Ehr. } \\
\text { coffaeiformis (A. Schm.) Cl. } \\
\text { didyma Ehr. } \\
\text { gorjanovichi (Pant.) Hust. } \\
\text { lineata (Donk) Cl. var.? } \\
\text { notabilis (Grev.) Cl. } \\
\text { ovalis } \text { (Hilse) Cl. } \\
\text { smithii (Bréb.) Cl. } \\
\text { sp. I } \\
\text { sp. II }\end{array}$ & + & $\begin{array}{l}\text { M,l,b } \\
\text { M,l,b } \\
\text { M,B,1,eu,b } \\
\text { B,b } \\
\text { M,l } \\
\text { M,1 } \\
\text { F,B } \\
\text { M,B,1,b,s,r }\end{array}$ & $\begin{array}{l}\otimes \\
\otimes \\
X \\
\otimes\end{array}$ & 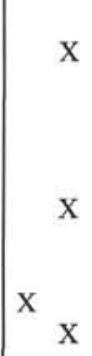 & & $\mathrm{X}$ & $\mathrm{X}$ & $\begin{array}{l}\text { Miocene - Recent, Med. } \\
\text { Miocene - Recent, Med. } \\
\text { Miocene - Recent } \\
\text { Miocene } \\
\text { Recent, Med. } \\
\text { Recent, Med. } \\
\text { Miocene - Recent } \\
\text { Lower-Miocene - Recent }\end{array}$ \\
\hline
\end{tabular}


TABLE 1 - Continued

\begin{tabular}{|c|c|c|c|c|c|c|}
\hline \multirow[b]{2}{*}{ Species } & \multirow[b]{2}{*}{ 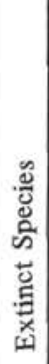 } & \multirow[b]{2}{*}{ Ecologies $^{\mathrm{a}}$} & \multicolumn{3}{|c|}{ Locality } & \multirow[b]{2}{*}{ Ages $^{b}$} \\
\hline & & & 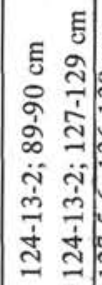 & 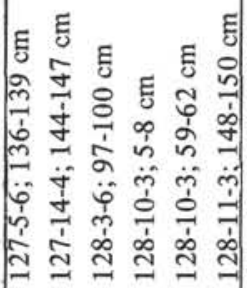 & 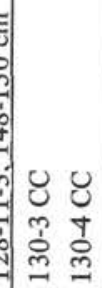 & \\
\hline $\begin{array}{l}\text { Epithemia } \\
\text { sorex Kütz. } \\
\text { turgida (Ehr.) Kütz. } \\
\text { zebra (Ehr.) Kütz. var. saxonica (Kütz.) Grun. }\end{array}$ & & $\begin{array}{l}\text { F,B,l,sw,e } \\
\text { F,B,eu,e } \\
\text { F,e }\end{array}$ & $\stackrel{\otimes}{\otimes}$ & & & $\begin{array}{l}\text { Pleistocene - Recent } \\
\text { Pleistocene - Recent } \\
\text { Miocene - Recent } \\
\end{array}$ \\
\hline Eunotia pectinalis (Kütz.) Rabh. & & $\mathrm{F}$ & & & 0 & Pleistocene - Recent \\
\hline $\begin{array}{l}\text { Fragilaria } \\
\text { construens (Ehr.) Grun. } \\
\text { hirosakiensis Kanaya } \\
\text { inflata (Heid.) Hust. } \\
\text { inflata (Heid.) Hust. var. istranfyi (Pant.) Hust. } \\
\text { leptostauron (Ehr.) Hust. } \\
\text { leptostauron (Ehr.) Hust. var. dubia Grun. }\end{array}$ & + & $\begin{array}{l}\mathrm{F}, 1, \mathrm{sw} . \\
\mathrm{M} \\
\mathrm{F} \\
\mathrm{F} \\
\mathrm{F}, \mathrm{b} \\
\mathrm{F}, \mathrm{b}\end{array}$ & & & & $\begin{array}{l}\text { Miocene - Recent } \\
\text { Miocene - ? } \\
\text { Fossil ? - Recent } \\
\text { Fossil? - Recent } \\
\text { Miocene - Recent } \\
\text { Miocene - Recent }\end{array}$ \\
\hline Fragilariopsis pliocena (Brun) Sheshuk & + & M & & $\mathrm{XX}$ & & Pliocene - ? \\
\hline Glyphodesmis williamsonii (W. sm.) Grun. & & $\mathrm{M}, 1, \mathrm{~b}$ & & $\mathrm{X}$ & & Recent, Med. \\
\hline Gomphonema sp. & & & $\mathrm{x}$ & & & \\
\hline $\begin{array}{l}\text { Grammatophora angulosa Ehr. } \\
\text { angulosa Ehr. var. islandica (Ehr.) Grun. } \\
\text { marina (Lyngb.) Kütz. var. adriatica Grun. } \\
\text { maxima Grun. } \\
\text { oceanica (Ehr.) Grun. var. macilenta (W. Sm.) Grun. } \\
\text { sp. I } \\
\text { sp. II } \\
\text { sp. III } \\
\text { cfr. stricta Ehr. var. fossilis Grun. } \\
\text { undulata Ehr. }\end{array}$ & + & $\begin{array}{l}\mathrm{M}, 1, \mathrm{e}, \mathrm{r} \\
\mathrm{M}, \mathrm{l}, \mathrm{e}, \mathrm{r} . \\
\mathrm{M}, \mathrm{l}, \mathrm{b}, \mathrm{r} \\
\mathrm{M}, \mathrm{l}, \mathrm{b} \\
\mathrm{M}, \mathrm{l}, \mathrm{b} \\
\mathrm{M}, \mathrm{b} \\
\mathrm{M}, \mathrm{b} \\
\mathrm{M}, \mathrm{b} \\
\mathrm{M}, \mathrm{b} \\
\mathrm{M}, \mathrm{l}, \mathrm{b}\end{array}$ & $\mathrm{x}$ & 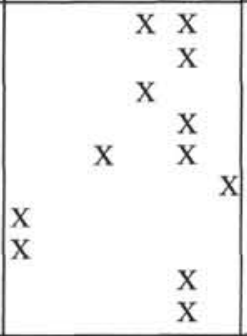 & & $\begin{array}{l}\text { Miocene - Recent, Med. } \\
\text { Miocene - Recent, Med. } \\
\text { Recent, Med. } \\
\text { Recent } \\
\text { Miocene - Recent } \\
\\
\text { Miocene - ? } \\
\text { Recent, Med. }\end{array}$ \\
\hline $\begin{array}{l}\text { Hyalodiscus } \\
\text { scoticus (Kütz.) Grun. } \\
\text { sp. }\end{array}$ & & $\begin{array}{l}\mathrm{M}, \mathrm{B}, \mathrm{l}, \mathrm{p} \\
\mathrm{M}, \mathrm{p}\end{array}$ & & $\mathrm{x}$ & & Miocene - Recent \\
\hline Isthmia sp. & & M & & $\mathrm{X}$ & & \\
\hline Liradiscus ovalis Grev. & + & $M$ & & & $\mathrm{X}$ & Eocene-Miocene - ? \\
\hline ?Liradiscus sp. & & $\mathrm{M}$ & & $\mathrm{X}$ & & \\
\hline $\begin{array}{l}\text { Mastogloia } \\
\text { brauni Grun. } \\
\text { cfr. elegans Lewis } \\
\text { elliptica (Ag.) Cl. var. dansei (Thw.) Cl. } \\
\text { sp. I } \\
\text { sp. II } \\
\text { sp. III }\end{array}$ & & $\begin{array}{l}\text { M,B,l,e } \\
\text { M,l,e } \\
\text { B,s,e } \\
\text { e } \\
\text { e } \\
\text { e }\end{array}$ & 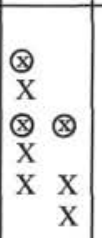 & & & $\begin{array}{l}\text { Recent } \\
\text { Recent, Med. } \\
\text { Pleistocene - Recent }\end{array}$ \\
\hline $\begin{array}{l}\text { Melosira } \\
\text { ambigua (Grun.) O. Müll. } \\
\text { granulata (Ehr.) Ralfs } \\
\text { granulata (Ehr.) Ralfs var. angustissima O. Mưll. } \\
\text { westii W. Sm. } \\
\end{array}$ & & $\begin{array}{l}\text { F,eu,p } \\
\text { F,eu,p } \\
\text { F,eu,p } \\
\text { M,n,l,p }\end{array}$ & & $\mathrm{X}$ & & $\begin{array}{l}\text { Miocene - Recent } \\
\text { Miocene - Recent } \\
\text { Miocene - Recent } \\
\text { Miocene - Recent }\end{array}$ \\
\hline $\begin{array}{l}\text { Navicula } \\
\text { comoides (Ag.?) Perag. } \\
\text { humerosa Bréb. } \\
\text { impercepta Hust. } \\
\text { pseudotuscula Hust. } \\
\text { radiosa Kütz. }\end{array}$ & & $\begin{array}{l}\text { M,l,p } \\
\text { M,l,eu,r } \\
\text { M,o,p } \\
\text { F } \\
\text { F }\end{array}$ & $x^{x}$ & $\mathrm{X}$ & & $\begin{array}{l}\text { Fossil ? - Recent } \\
\text { Miocene - Recent } \\
\text { Fossil ? - Recent } \\
\text { Recent } \\
\text { Miocene - Recent }\end{array}$ \\
\hline ?Navicula sp. & & & & $\mathrm{X}$ & & \\
\hline
\end{tabular}


TABLE 1 - Continued

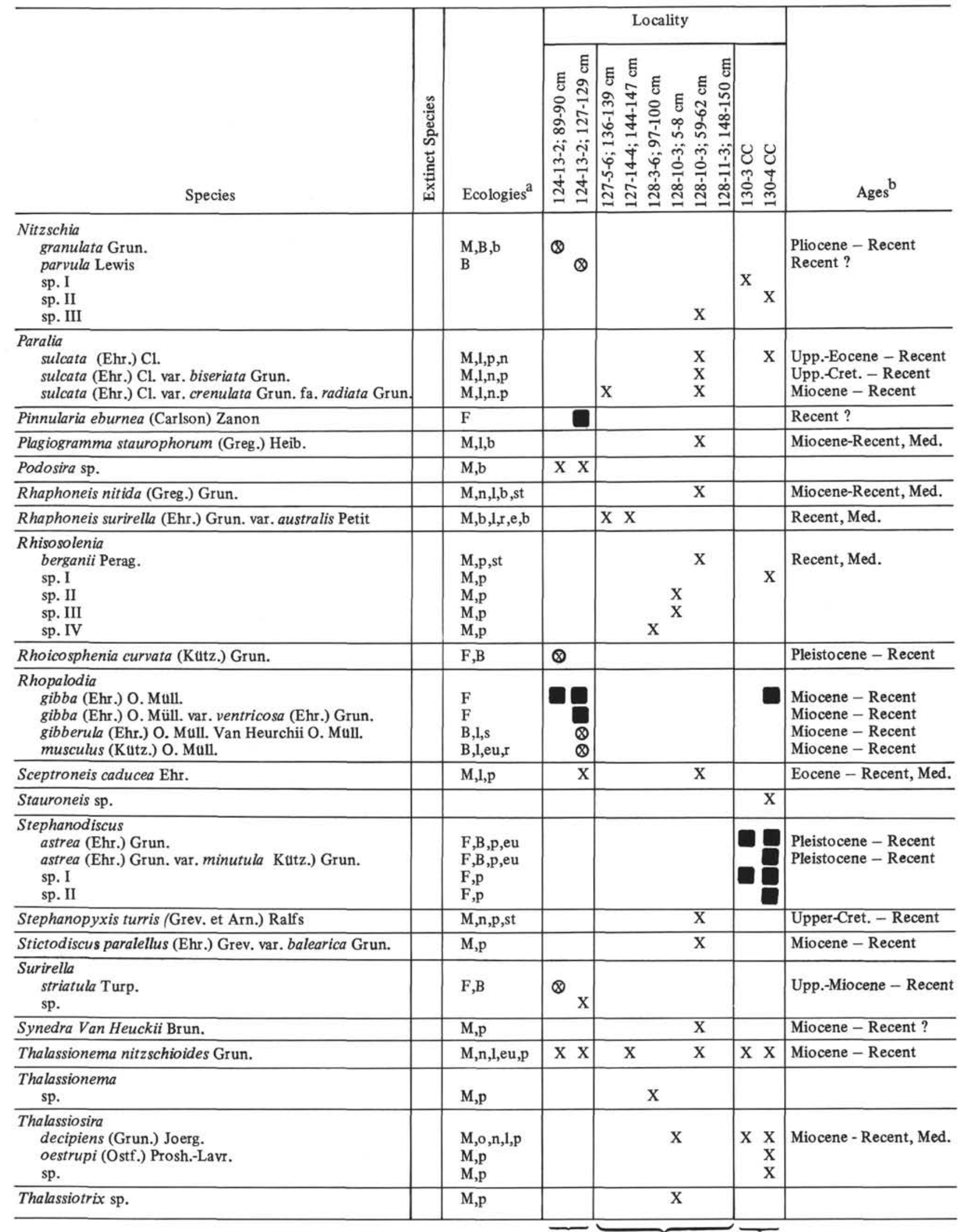


TABLE 1 - Continued

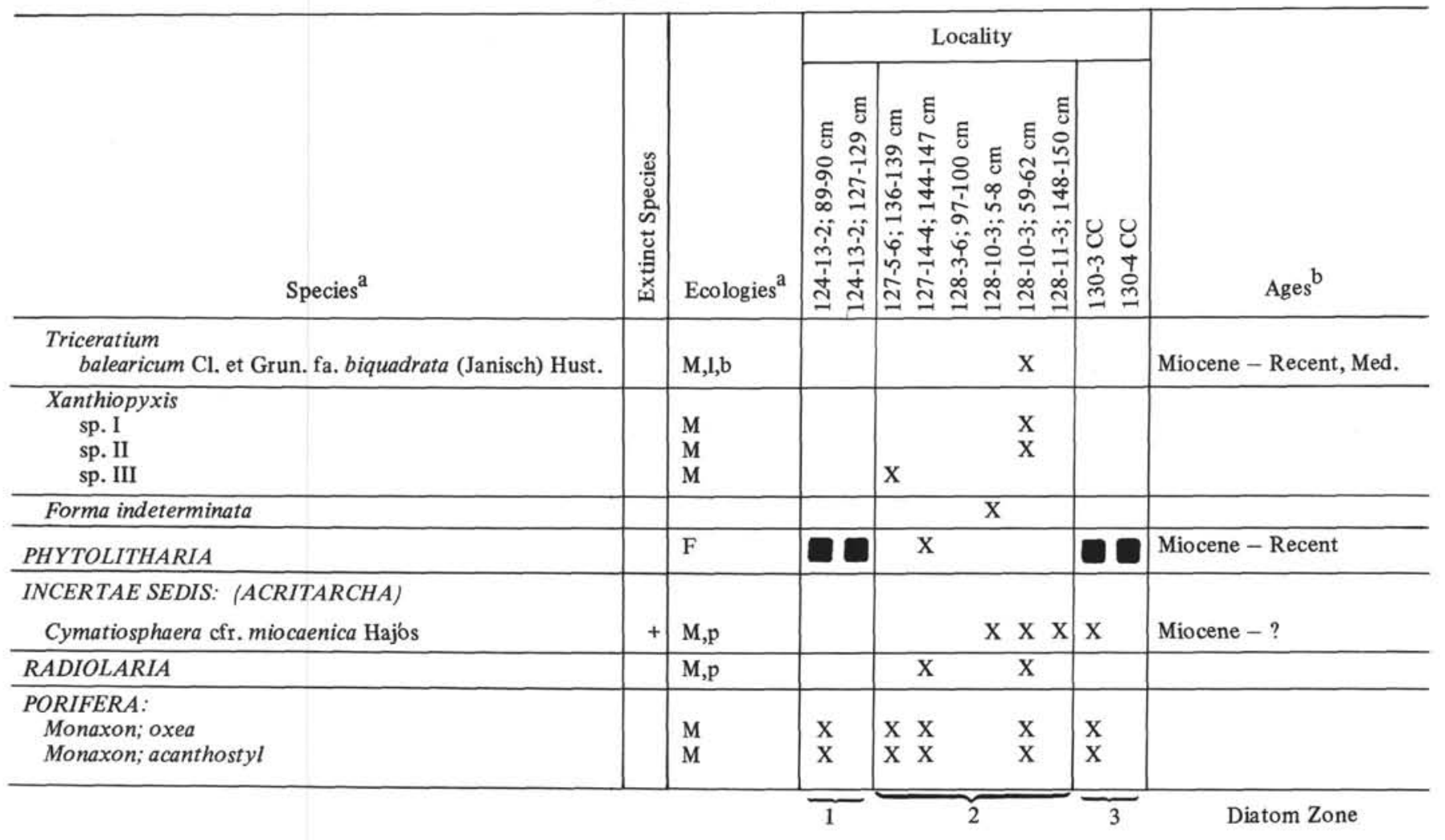

\footnotetext{
${ }^{\mathrm{a}}$ Explanation of Ecological Remarks: $\mathrm{M}=$ Marine species $\mathrm{X} ; \mathrm{B}=$ Brackish water species $\otimes ; \mathrm{F}=$ Fresh water species $\mathrm{O}$; $=$ Oceanic; $\mathrm{n}-\mathrm{Neritic}$; $1=$ Littoral; $\mathrm{r}=$ River mouths; $\mathrm{sw}=$ Stagnant water $\mathrm{s}=$ Salina $; \mathrm{p}=$ Planktonic; $\mathrm{e}=$ Epiphytic; $\mathrm{b}=$ Benthonic; eu - Euryhaline; st - Stenothermal; sh $=$ Stenohaline

${ }^{\mathrm{b}}$ Med. = Mediterranean
}

Cyclotella sp.

Diatomella balfouriana (epiphytic)

Fragilaria leptostaurota (benthonic)

Fragilaria leptostaurota (planktonic euryhaline)

Pinnularia eburnea

Rhiocosphenia curvata

Rhopalodia gibba

Rhopalodia gibba var. ventricosa.

Autochthonous brackish-water and freshwater forms indicate the presence of a shallow-water, land-locked, littoral area which did not undergo a significant change in water depth before the advent of later geological events. On the basis of a few extinct fossil species such as Archaeomonas californica, A. japonica, A. melo, Mesocena elliptica var. pentagona, Denticula hustedtii, Diploneis gorjanovicii, which have so far been recorded from Miocene (Tortonian-Sarmatian) deposits only, the sediment can be dated as late Miocene.

\section{Division 2 - Sapropels from the Hellenic Trench}

On the basis of the floral assemblage, sediments of the samples from Sites 127 and $128(127-5-6,136-139 \mathrm{~cm}$; 127-14-4, 144-147 cm; 128-3-6, 97-100 cm; 128-10-3, 5-8 $\mathrm{cm} ; 128-10-3,59-62 \mathrm{~cm} ; 128-11-3,148-150 \mathrm{~cm}$ ) were formed approximately contemporaneously and under the same environmental conditions. The samples of these two sites can be correlated. The floral spectrum consists of (1) purely marine assemblages, showing the predominance of Silicoflagellata which require an open sea environment of normal salinity, and (2) neritic, littoral planktonic diatoms. The number of oceanic and stenohaline planktonic forms is also considerable. The species Cannopilus sphaericus indicates a salinity higher than $30 \%$. Subtropical and tropical stenothermal species favoring warm waters are abundant. The species Treceratium balearicum var. biquadrata, Stictodiscus parallelus var. balearica and Bacteriastrum comosum are known in Europe to occur exclusively in the region of the Balearic Islands.

Oceanic warm-water forms:

Asteromphalus robustus (stenohaline)

Coscinodiscus curvatulus

Coscinodiscus divisus

Coscinodiscus lineatus

Coscinodiscus marginatus (stenohaline)

Coscinodiscus nitidus

Coscinodiscus oculus-iridis (stenohaline)

Rhisosolenia bergonii

Thalassiosira decipiens

Bacteriastrum comosum is a marine, neritic (stenohaline) species. As implied by the known salinity ranges of the species, the salinity of the environment must have been higher than 20 to $30 \%$ and $30 \%$, respectively. 
Stenothermal warm-water forms, indicating a warm to temperate sedimentary environment, include the following: Actinoptychus senarius

Cocconeis ornata var. signata

Rhaphoneis nitida

Rhisosolenia bergonii

Stephanopyxis turris

Besides the warm-to-temperate-water-favoring species of Rhisosolenia, Bacteriastrum and Coscinodiscus, the representatives of Acritarchs, Radiolaria and Silicospongia are characteristic.

On the basis of the floral assemblage the age of the sediment is early Quaternary.

\section{Division 3 - Blue to Black Clays from the Mediterranean Ridge}

The floral assemblages of Samples 130-3-CC and 130-4-CC of Site 13 are the same and indicate that the sediment is marine. However, along with marine and littoral planktonic forms, considerable numbers of fresh to brackish water, euryhaline planktonic forms are found. Among the marine planktonic forms the species Coscinodiscus excentricus and its varieties, which frequently occur in river mouths, are characteristic. The fresh and brackish water planktonic forms are presumably allochthonous and most likely have been introduced into the Mediterranean with the sediment of the Nile River delta. Most of the identified species of well-known stratigraphic range are still-living forms. The age of the sediment seems to be Quaternary.

On the basis of the studied samples, the paleogeographic distribution of the species has also been examined. The extinct species were found to show very little affinity, if any, with the forms occurring in the Miocene-Pliocene sediments of the peri-Mediterranean countries. A considerable number of the species presently live in the Mediterranean Sea. Many of the oceanic neritic and littoral species are known to occur on the Atlantic coast of Europe and on the Pacific coastal zones of America and Asia, as well as in the subtropical and tropical areas of the southern seas. Least affinity is shown with the known taxa of the Indian Ocean by both the fossil and recent species. At any rate, in Pliocene and Quaternary times the region of the Mediterranean Sea must have had a westward connection with the open ocean.

\section{REFERENCES}

Brun, J., 1891. Diatomées espèces nouvelles, marines, fossiles et pélagiques. Mém. Soc, Phys. Hist. Nat. de Genève, T. 31 (1), 47 p, pls. 11-12.

Brun, J. and Tempère, I., 1889. Diatomées fossiles du Japon. Mém. Soc. Phys. Hist. Nat. de Genève, T. 30 (9), 75 p, pls. 1-9.

Cholnoky, B., 1959. Neue und seltene Diatomeen aus Afrika. IV. Diatomeen aus der Kaap-Provinz. Österreichische Botanische Zeitschrift. 106. 69 p, 9 Textabl. figs. 1-352.

Cleve-Euler, A., 1951-1955. Die Diatomeen von Schweden und Finnland. Kungl. Svenska Vet. Akad. Handlingar, Fjärde Serien Bd. 2. No. 1. Teil. I. 1951. pp. 1-163. T. I-VI. Fig. 1-294; Bd. 4. No. 1. Teil II. 1953. pp. 1-158.
Fig. 292-483; Bd. 4. No. 5. Teil 1953. pp. 1-255. Fig. 484-970; Bd. 5. No. 4. Teil IV. 1955. pp. 1-232. Fig. 971-1306, 3 (3), Teil V. 1952. pp. 1-153. Fig. 1318-1583. Taf. VII.

Dahm, H. D., 1956. Diatomeenuntersuchungen zur Geschichte der westlichen Ostrsee. Meyniana. 5, 7-50, Tab. 1-2, Taf. 1-3, Abb. 1-11.

1963. Diagnose von Brackvassersedimenten mit Hilfe der Diatomeen. Fortschr. Geol. Rheinld. Westf., 10, pp. 95-106, Abb. 1-4.

Deflendre G. and Delflendre-Rigaud, M., 1969. Nannofossiles siliceux I. Archaeombnadaceae. Fichier Micropaleontologique General. Série 19. Centre National de la Recherche Scientifique. pp. I-IX. pls. 4173-4400. Paris.

Gleser, S. J., 1966. Silicoflagellatophyceae. Flora plantarum Cryptogamarum URSS. VII. 1-330. figs 1-28. pls. 1-XXXIII.

Greville, R. K., 1865. Description of New and Rare Diatoms. Series XIV. Transactions of the Microscopical Society of London. XIII. (New series) 1-105. pls. I-IX.

Gemeinhardt, K., 1930. Silicoflagellatae. In. Rabenhorst, L. Kryptogamen. Flora, 10. 1-87, Figs. 1-69, T. 1.

Grunow, A., 1884. Die Diatomeen von Franz-Josefs Land. Denkschr. d. Akad. d. Wiss. Math.-Nat. Class. 48 (Abt. 2), 53-112, T. 1-5.

Hajós, M., 1959. Die Algen der Kieselgurschichten von Szurdokpüspöki. Bull. Hungarian Geol. Soc., 89 ( $\left.{ }^{\circ} 2\right)$, 155-169, pls. 4-6. 5 figs.

, 1962. Holozäne Kieselgurschichten bei Ujpest/ Budapest/. Bull. Hungarian Geol. Soc. $92 \mathrm{n}^{\mathrm{d}}$ 4), 464-466, 1 fig.

1964. Geologische und Paleobotanische Untersuchung der Miozänen Diatomenführenden Sedimente am Fusse des Mátra-Gebirges. Annual Report Hungarian Geol. Inst., 162, pp. 495-510.

Hajós M., 1966. Das Mikroplankton der Kieselgurschichten im Miozän des Mecsekgebirges. Annual Report Hungarian Geol. Inst., 1964. pp. 139-171, 26 figs. 5 pls.

1968. Die Diatomeen der Miozänen Ablagerungen des Mátravorlandes. Geologica Hungarica-Series Paleontologica. 37, 1-401, 27 figs. 68 pls.

1968/a. Neogene Phytolitharien aus Ungarn. Annual Report Hungarian Geol. Inst., 1966. pp. 225-240, 4 pls.

1970. Microflora of the Lower Pannonian diatom-bearing beds of the Neogene basin of Csákvár. Annual Report Hungarian Geol. Inst., 1968, pp. 33-48, 4 pls.

1971. Methods for studying siliceous Microfossils and their Geological and Stratigraphic significance in Hungary. Proc. II Planktonic Conf., Roma 1970. pp. 599-606, fig. 1., tables 1-3.

1971. La microflore des formations a diatomites sarmatiennes de la région orientale de la Montagne de Mecsek. 5 éme Congress International du Neogene Mediterraneen. Lyon 1971. (in press)

Hanna, G. D. and Grant, W. M., 1929. Brackish-water Pliocene Diatoms from the Etchegoin Formation of Central California. J. Pal. 3 (1), 87-100, pls, 11-14.

Hendey, N. I., 1964. An Introductory Account of the Smaller Algae of British Coastal Waters. Part V. Bacillariophyceae/Diatoms/. Fishery Investigations. Series IV.

Hustedt, F., 1927-1964. Die Kieselalgen Deutschlands, Österreichs und der Schweiz. In: Rabenhorst, L. 
Kryptogamen-Flora, 7 (Teil 1), 1-920, Figs. 1-542; Teil 2 , pp. 1-845, Figs. 543-1179; Teil 3, pp. 1-556, Figs. 1180-1591.

Ichikawa, W, Fuji, N. and Bachmann, A., 1964. Fossil Diatoms, Pollen Grains and Spores, Silicoflagellates, and Archaeomonads in the Miocene Hojuji Diatomaceous Mudstone, Noto Peninsula, Central Japan. Science Reports of Kanazawa University, IX. (1-2), 25-118, T. $\mathrm{I}-\mathrm{XIII}+\mathrm{I}-\mathrm{VII}$.

Loeblich, A. R., Loeblich, L. A. and Tappan, H., 1968. Annotated Index of Fossil and Recent Silicoflagellates and Ebridians, Geol. Soc. Am. pp. 1-319, pls. 1-53.

Lohman, E. E., 1938. Pliocene Diatoms from the Kettleman Hills, California. Geol. Surv. Prof. Paper, 189-C, pp. 81-102, pls. 20-23.

1939. Pleistocene Diatoms from Long Island, New York. Geol. Surv. Prof. Paper, 189-H, pp. 229-237.

Mertz, D., 1966. Mikropaläontologische und Sedimentologische Untersuchung der Pisco-Formation. Südperus. Palaeontographica Abt. B. Bd. 118. Lief. 1-3. pp. 1-51, pls. 1-7.

Pantocsek, J., 1886-1905. Beiträge zur Kenntnis der fossilen Bacillarien Ungarns. I. pp. 1-74, T. 1-30, figs. $1-320,1886$; II. pp. $1-123$, T. 1-30, figs. 1-129, 1889 ; III. T. $1-42$, figs. $1-584,1892$. pp. $1-118,1905$. Nagytapolcsány-Pozsony.

Reinhold, Th., 1937. Fossil Diatoms of the Neogene of Java and their Zonal Distribution. Verh. Geol. Mijnbouv. Gen. von Nederland en Kol. Geol. Ser. 12, pp. 43-133, pls. 1-21. Gravenhage.

Ross, R., 1963. The Diatom Genus Carpatogramma and the Identity of Schizostauron. Bull. British Museum (Natural History) Botany. 3 (2), 47-92. Textfigs. 1-31, pls. 1-2.

Sheshukova-Poretzkaya, V. S., 1967. Neogenovye morskye diatomovye vodorosfi Sachalina Kamchatki pp. 1-432. pls. 1-50. Leningrad.

Simonsen, R. and Kanay, T., 1961. Notes on the Marine Species of the Diatom genus Denticula Kütz. Int. Revue ges. Hydrobiol. 46 (4) 498-513. Textfig. 1. pl. 1.
Stradner, H., 1961. Uber fossile Silicoflagelliden und die Möglichkeit ihrer Verwendung in der Erdölstratigraphie. Erdöl und Kohle, Jhg. 14, Nr. 2. pp. 87-92. Abb. 1, T. 1-3.

Tynan, E. J., 1957. Silicoflagellates of the Calvert formation (Miocene) of Maryland. Micropaleontology, 3 (2), 127-136, pl. 1. Textfigs. 1-3.

Van Heurck, H., 1896. A Treatise on the Diatomaceae. pp. 1-558. pls. 1-35, Textfigs. 1-291.

Wornardt, W. W., 1964. Pleistocene Diatoms from Mono vand Panamint Lake Basins, California. Occ. Papers of the Calif. Acad. of Sci., No. 46, pp. 1-27, figs. 1-2, pls. $1-2$.

Zanon, V., 1934. Silicoflagellate fossili Italiane. Acta P. Acad. Sc. Nov. Lync., Anno 87, Sers. I. pp. 1-44. pl. 1.

\section{Other References Not Cited, But Pertinent}

Brelie, G., 1956. Diatomeen als Fazies-Fossilien. Geologische Rundschau. 45 (1), 84-97. Abb. 1-2. Tab. 1-3. Stuttgart.

Brockmann, Chr., 1940. Diatomeen als Leitfossilien in Küstenablagerungen. Wesküste Arc. für Forshung Technik und Verwaltung in Marsch und Wattenmeer, Zweiter Jahrgang Doppelheft 2/3 pp. 151-181. Abb. 1-9. Heide J. Holst.

Hays, J. D., Saito, T. et al., 1969. Pliocene-Pleistocene Sediments of the Equatorial Pacific: Their Paleomagnetic, Biostratigraphic, and Climatic Record. Bull. Geol. Soc. Am. 80, 1481-1514, figs. 1-16. pl. 1 .

Hendey, N. I., 1958. Marine Diatoms from some West African Ports. Journ. R. Micr. Soc. Ser. III. LXXVII. Parts 1-2. pp. 28-85. Pls. I-VI.

Hopkins, D. M., Scholl, D. W. et al., 1969. Cretaceous, Tertiary, and Early Pleistocene Rocks from the Continental Margin in the Bering Sea. Bull. Geol. Soc. Am. $80\left(\mathrm{~N}^{\circ} 8\right), 1471-1480$, figs. 1-2, pls. $1-2$.

Kolbe, R. W., 1954. Diatoms from Equatorial Pacific Cores. Reports of the Swedish Deep-Sea Expedition 1947-1948. 6 (1), 1-49. pls. 1-4. 
PLATE 1

Magnifications $\times 1000$

Scale bar represents 10 microns

Figure 1 Archaeomonas mangini Deflandre

Figures 2, 3, 5 Archaeomonas japonica Deflandre

Figures 4, 6 Archaeomonas inconspicua Deflandre

Figure 7 Archaeomonas melo Deflandre

Figure 8 Mesocena elliptica (Ehrenberg) Ehrenberg var. quadrangula Bachmann \& Ichikawa.

Figures 9, $10 \quad$ Dictyocha fibula Ehrenberg

Figures 11, 12 Dictyocha speculum Ehrenberg

Figure 13 Hermesinum cfr. adriaticum Zach.

Figures 14, 15 Melosira granulata (Ehrenberg) Ralfs

Figure 16 Melosira granulata (Ehrenberg) Ralfs var. angustissima O. Müller.

Figures 17, 18 Melosira granulata (Ehrenberg) Ralfs

Figure 19 Melosira westii W. Smith

Figures 20, $21 \quad$ Paralia sulcata (Ehrenberg) Cleve

Figure $22 \quad$ Cyclotella comta (Ehrenberg) Kützing

Figures 23,24 Cyclotella sp. 
PLATE 1
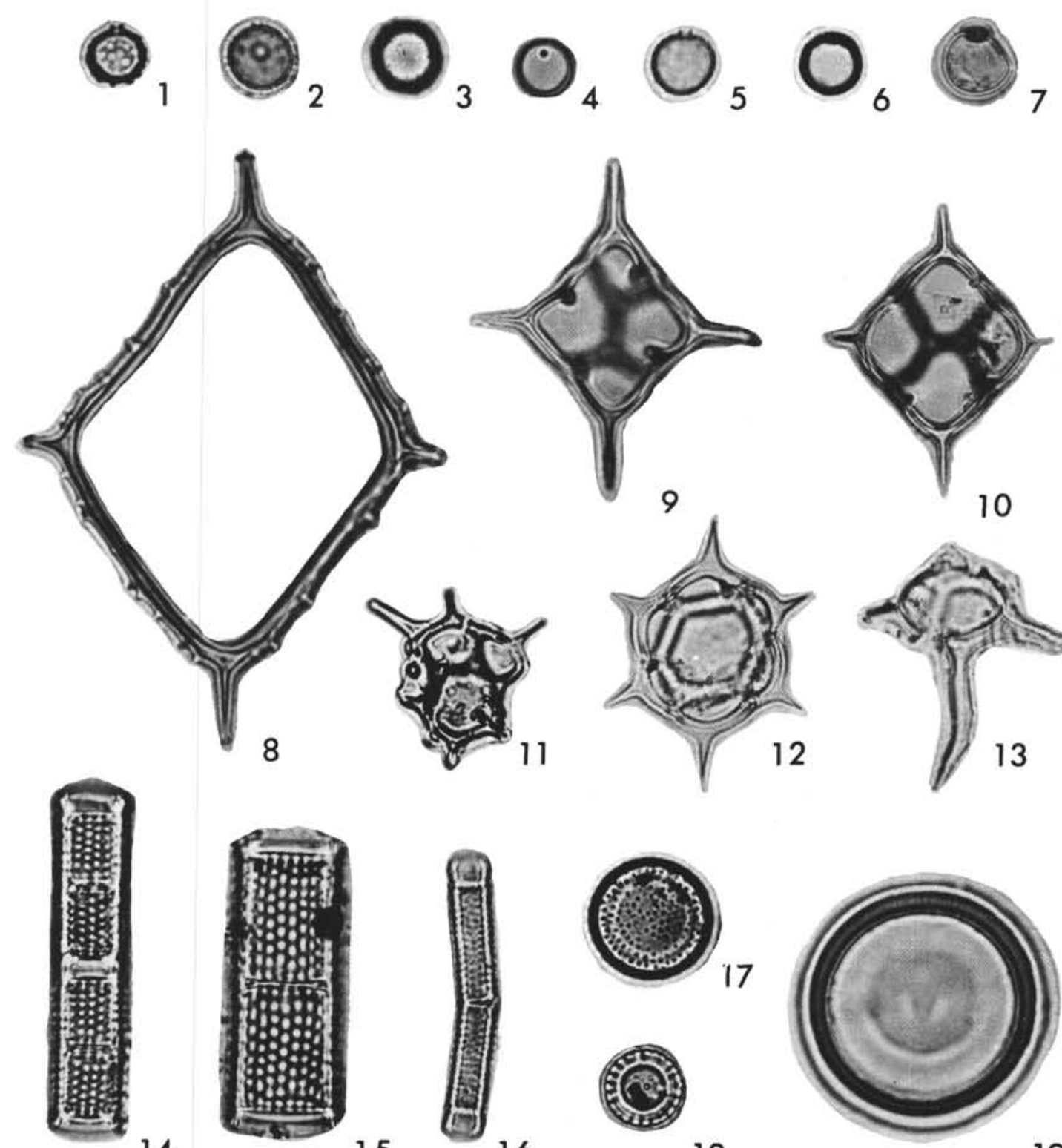

14
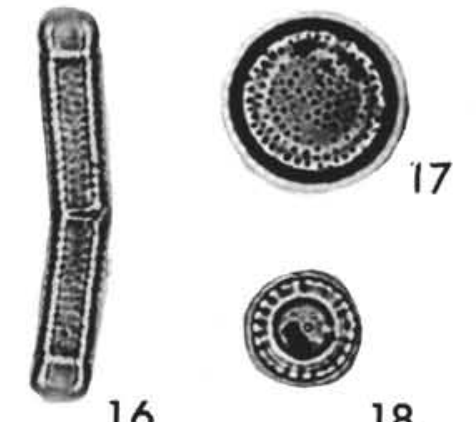

18
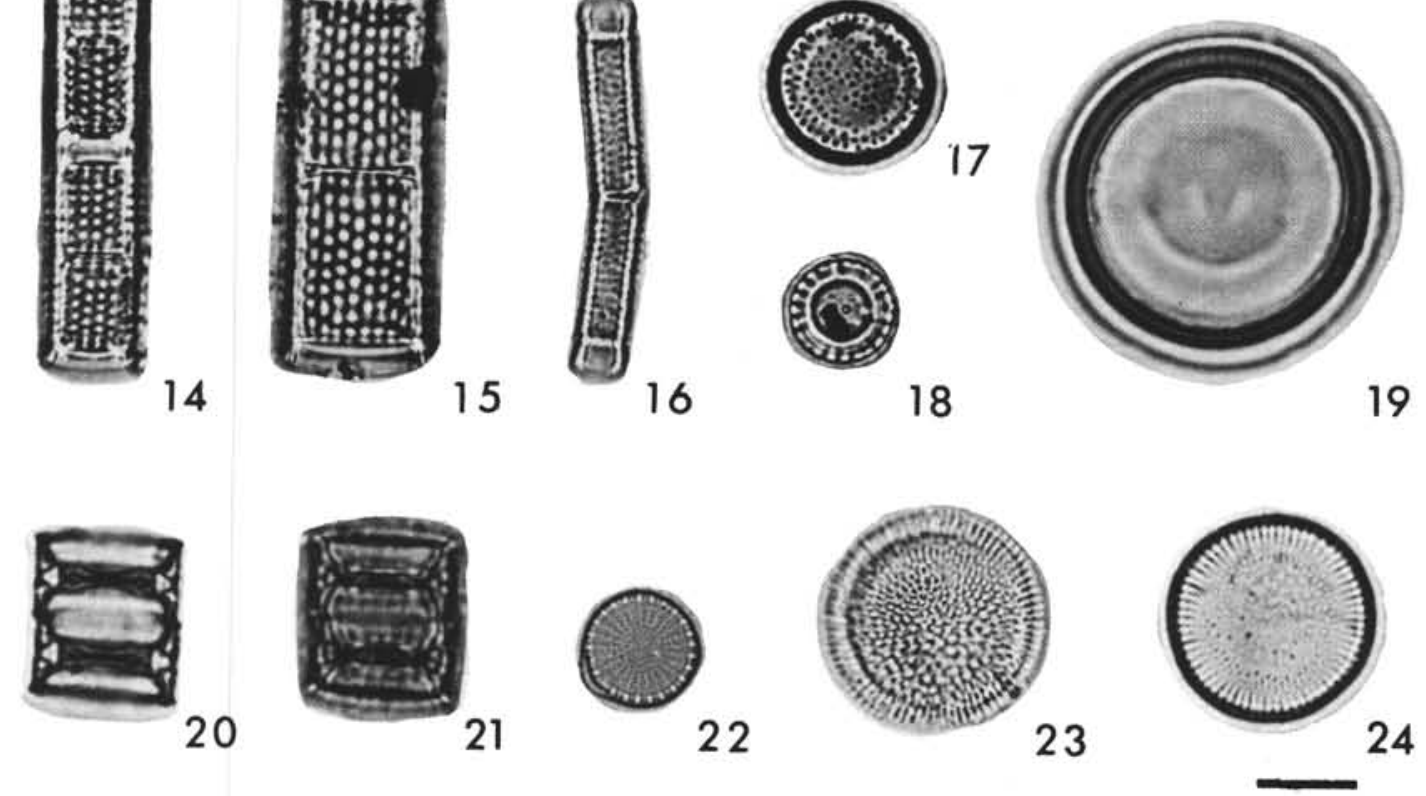
PLATE 2

Magnifications $\times 1000$

Scale bar represents 10 microns

Figure 1

Figures 2, 3

Figure 4

Figure 5

Figure 6

Figures 7, 8

Figures 9, 10

Figures 11, 12, 1

Figures 13, 14

Figures 15, 16

Figure 17, 18
Paralia sulcata (Ehrenberg) Cl. var. biseriata Grunow

Podosira sp.

Hyalodiscus scoticus (Kützing) Grunow

Hyalodiscus sp.

Stephanopyxis turris (Greville Arnott) Ralfs

Thalassiosira oestrupi (Ostenfeld) Proskina-Lavrenko

Thalassiosira decipiens (Grunow) Joergensen

Stephanodiscus astrea (Ehrenberg) Grunow

Stephanodiscus astrea (Ehrenberg) Grunow var. minutula (Kützing) Grunow

Coscinodiscus excentricus Ehrenberg var. micropora Grunow

Coscinodiscus curvatulus Grunow var. macropunctata Hajós 
PLATE 2
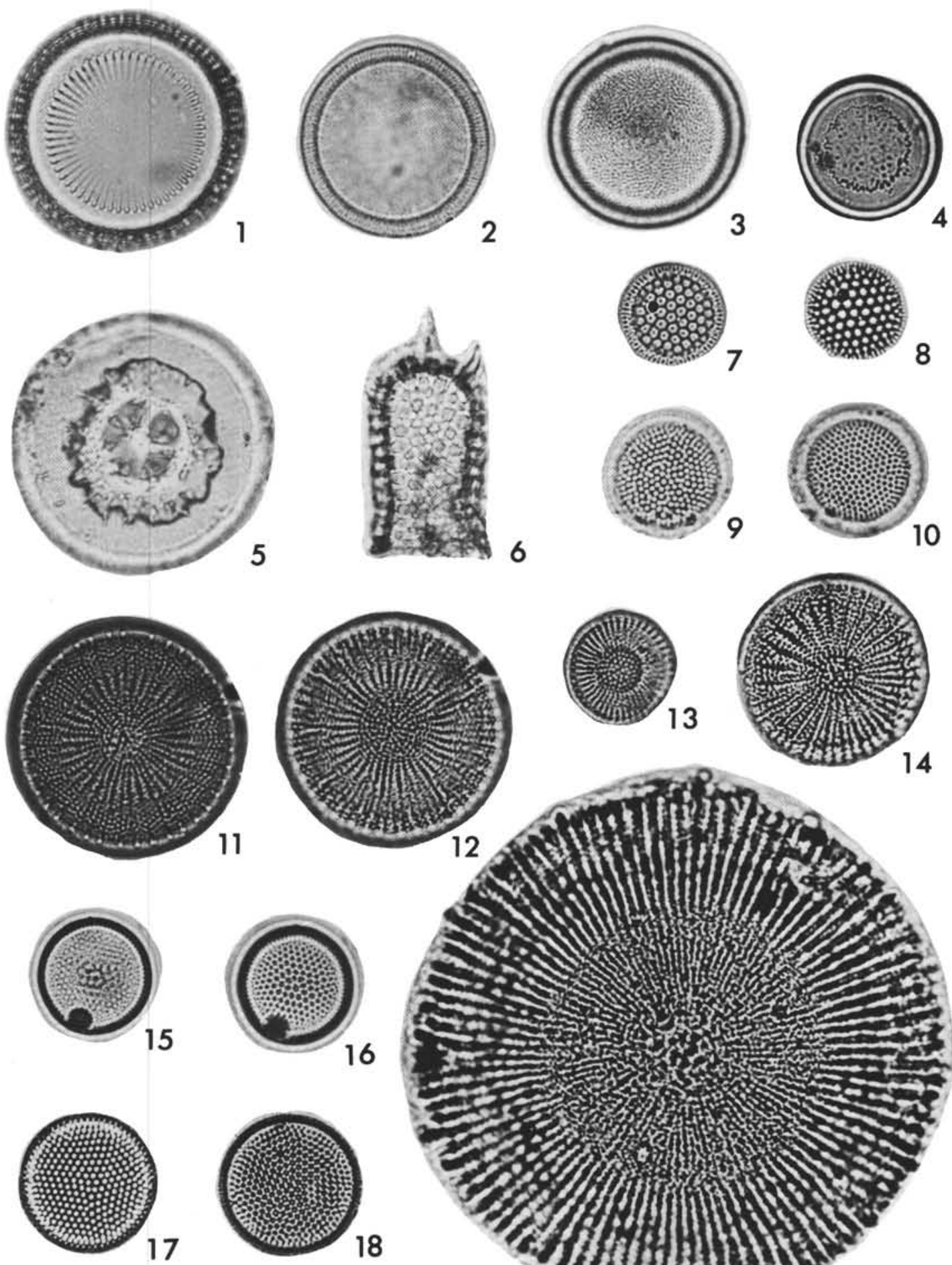

18

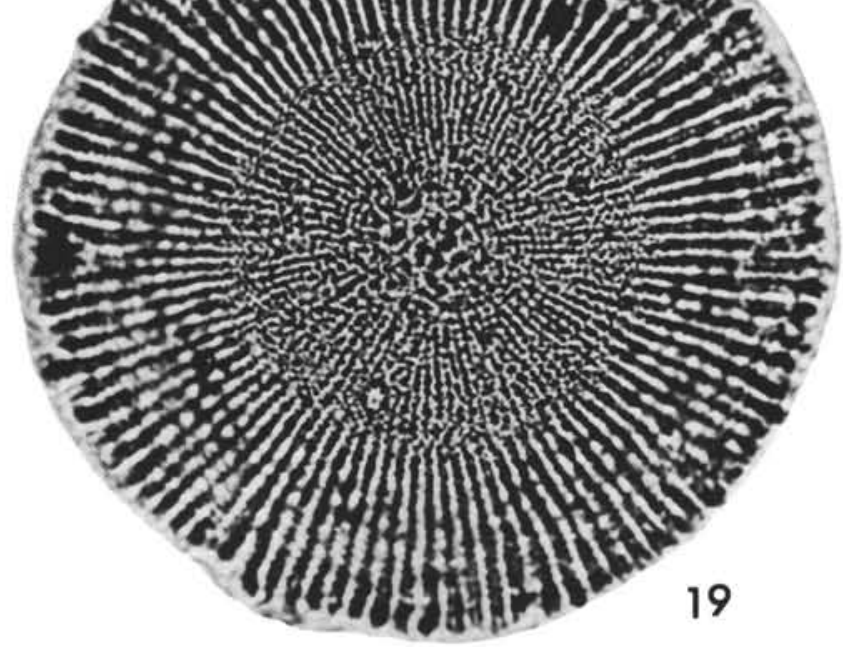




\section{PLATE 3}

Magnifications $\times 1000$

Scale bar represents 10 microns

Figures 1,2,5 Coscinodiscus oculus-iridis Ehrenberg

Figures 3, 4, 10 Coscinodiscus excentricus Ehrenberg

Figure $6 \quad$ Coscinodiscus nitidus Gregory

Figure $7 \quad$ Coscinodiscus marginatus Ehrenberg

Figures 8,9 Coscinodiscus hustedtii Jousé 
PLATE 3
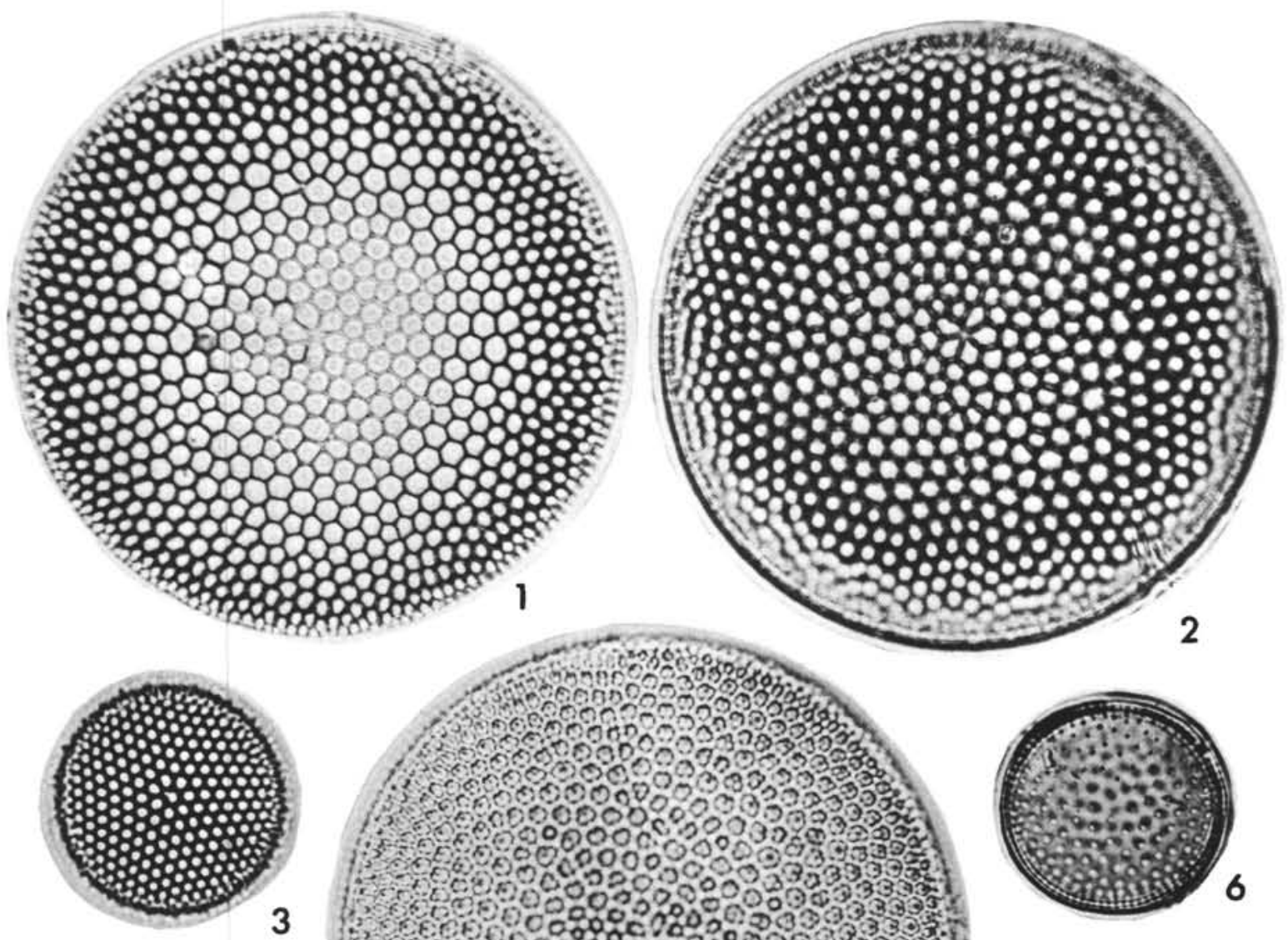

$2008060 \%$
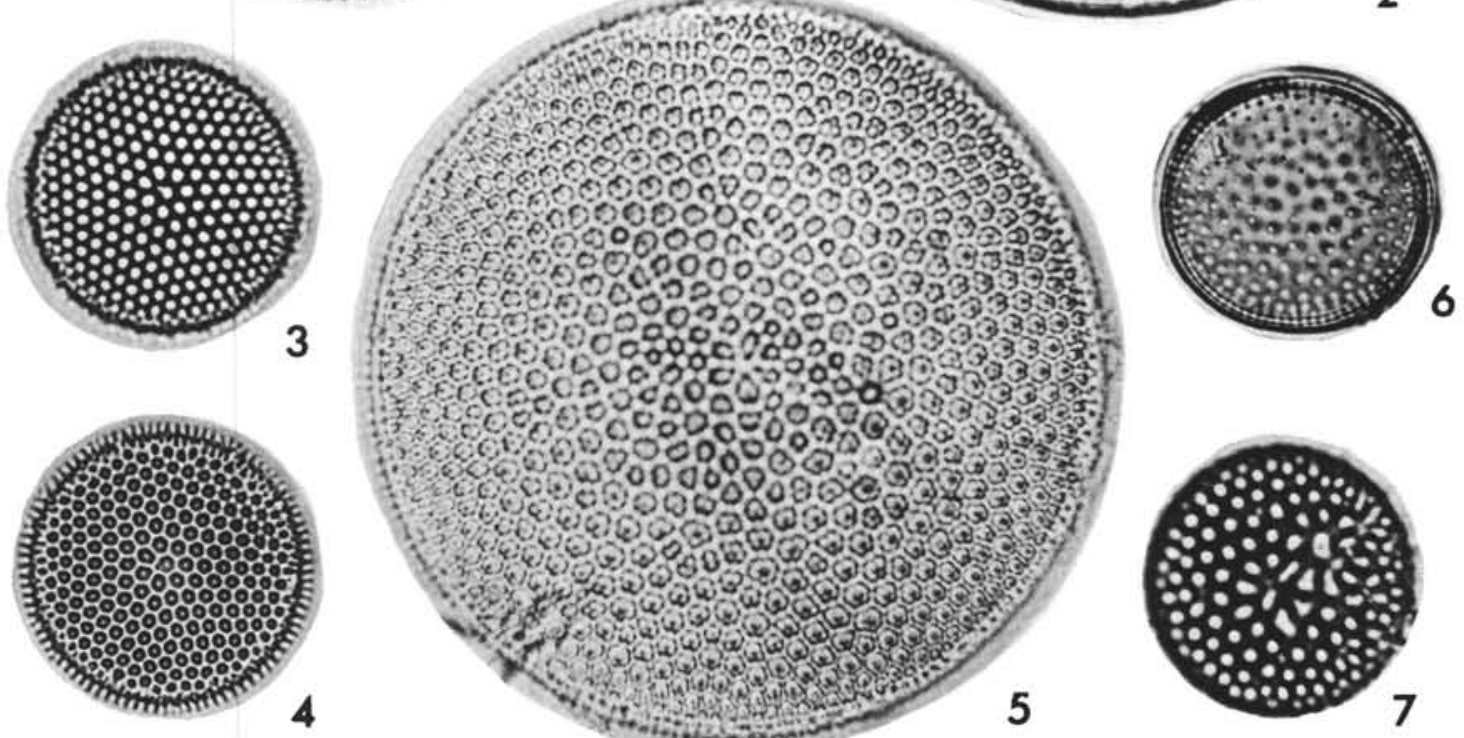

$3.10000000 \% 200303$
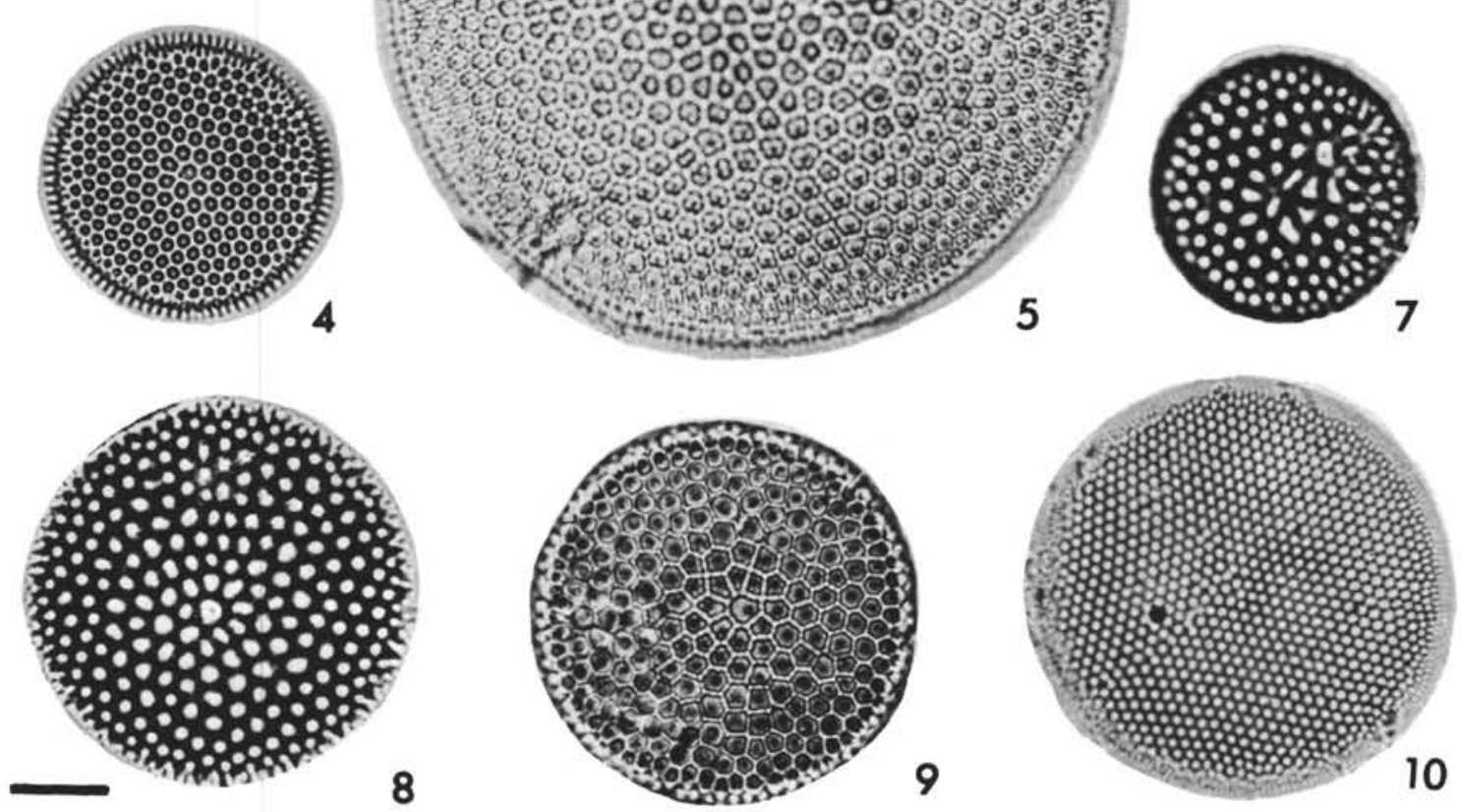


\section{PLATE 4}

Magnifications X 1000 Scale bar represents 10 microns

Figures 1, 2, 4, Coscinodiscus excentricus Ehrenberg

Figures 3, $5 \quad$ Coscinodiscus kützingii A. Schmidt

Figures 6,7 Coscinodiscus divisus Grunow

Figures 8,9 Coscinodiscus curvatulus Grunow var. arcuata Hajós

Figure 10 Stictodiscus paralellus (Ehrenberg) Greville var. balearica Grunow

Figure 11 Actinoptychus reinholdii Hajós 


\section{PLATE 4}
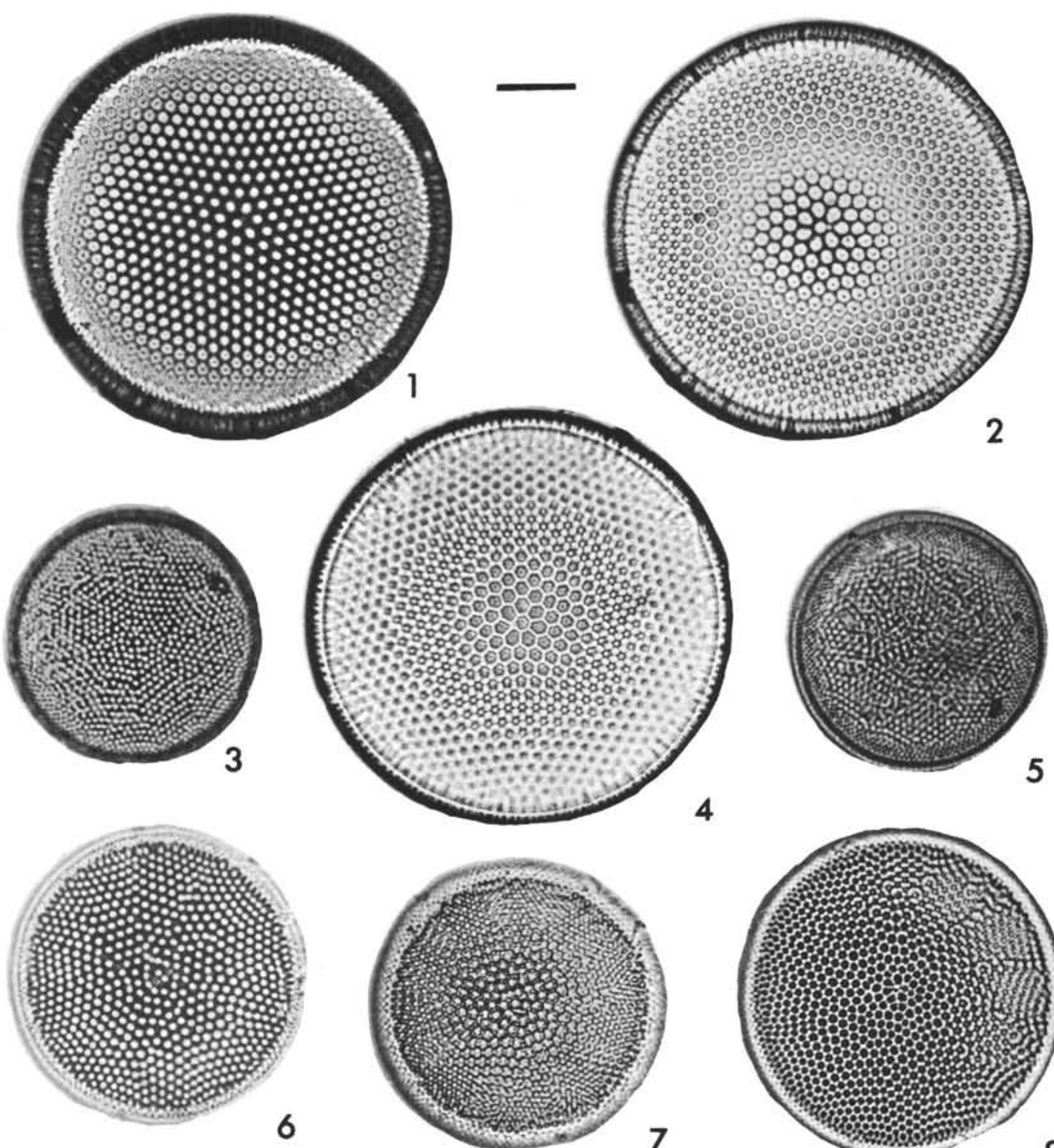

4
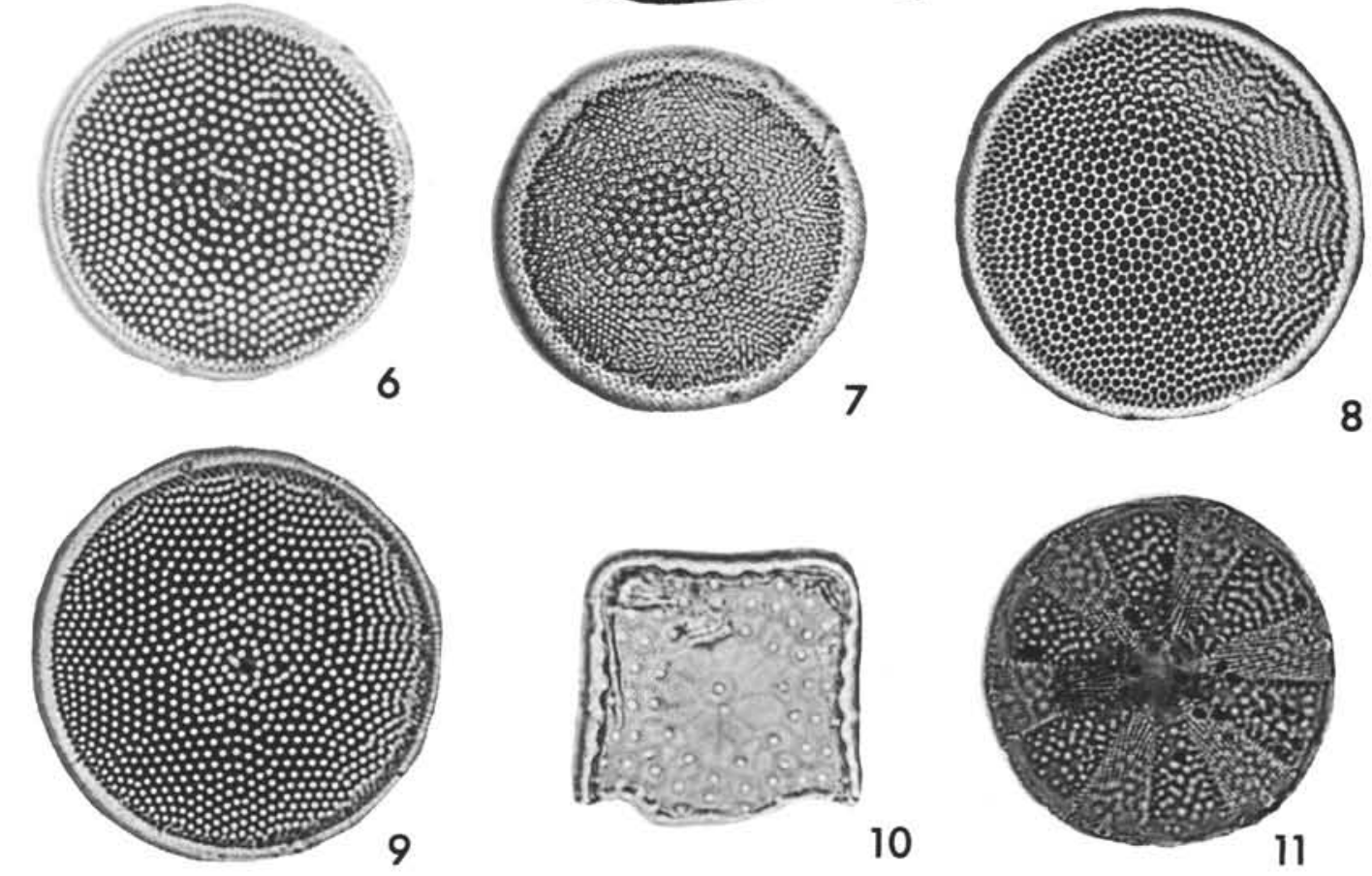


\section{PLATE 5}

Magnifications $\times 1000$

Scale bar represents 10 microns

Figure $1 \quad$ Asteromphalus robustus Castracane

Figures 2, $3 \quad$ Actinoptychus senarius (Ehrenberg) Ehrenberg

Figures 4, 5, 10 Actinocyclus octonarius Ehrenberg var. crassa (W. Smith) Hustedt

Figure 6 Actinocyclus octonarius Ehrenberg var. minuta (Pantocsek) Hajós

Figures 7,9 Actinocyclus octonarius Ehrenberg var. tenella (Brebisson) Hajós

Figure $8 \quad$ Actinocyclus octonarius Ehrenberg 
PLATE 5
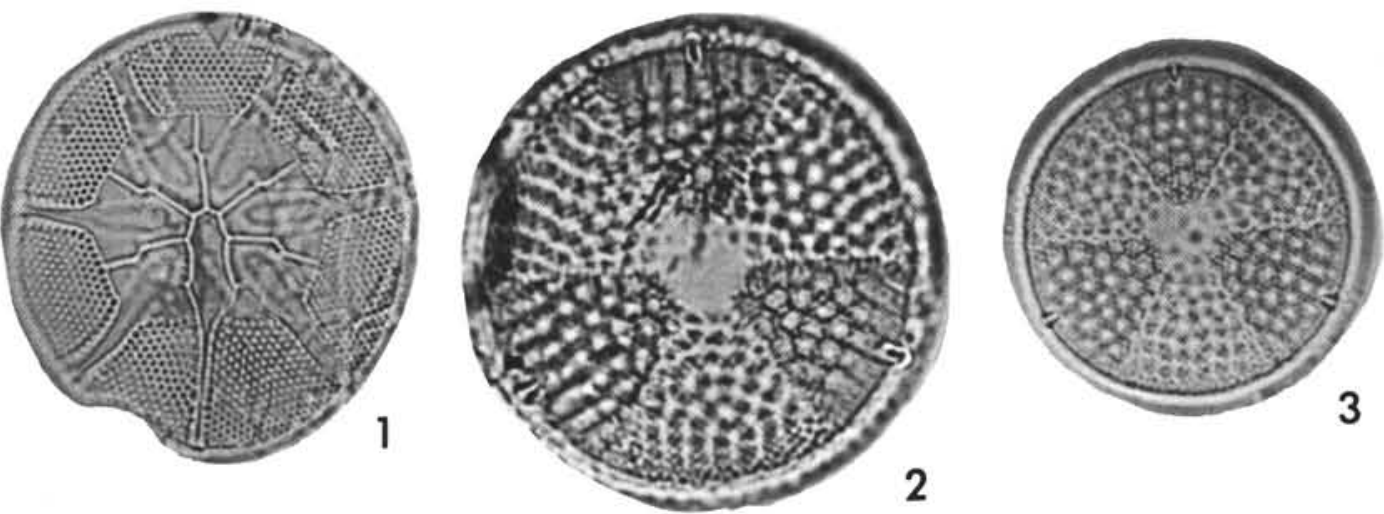

2
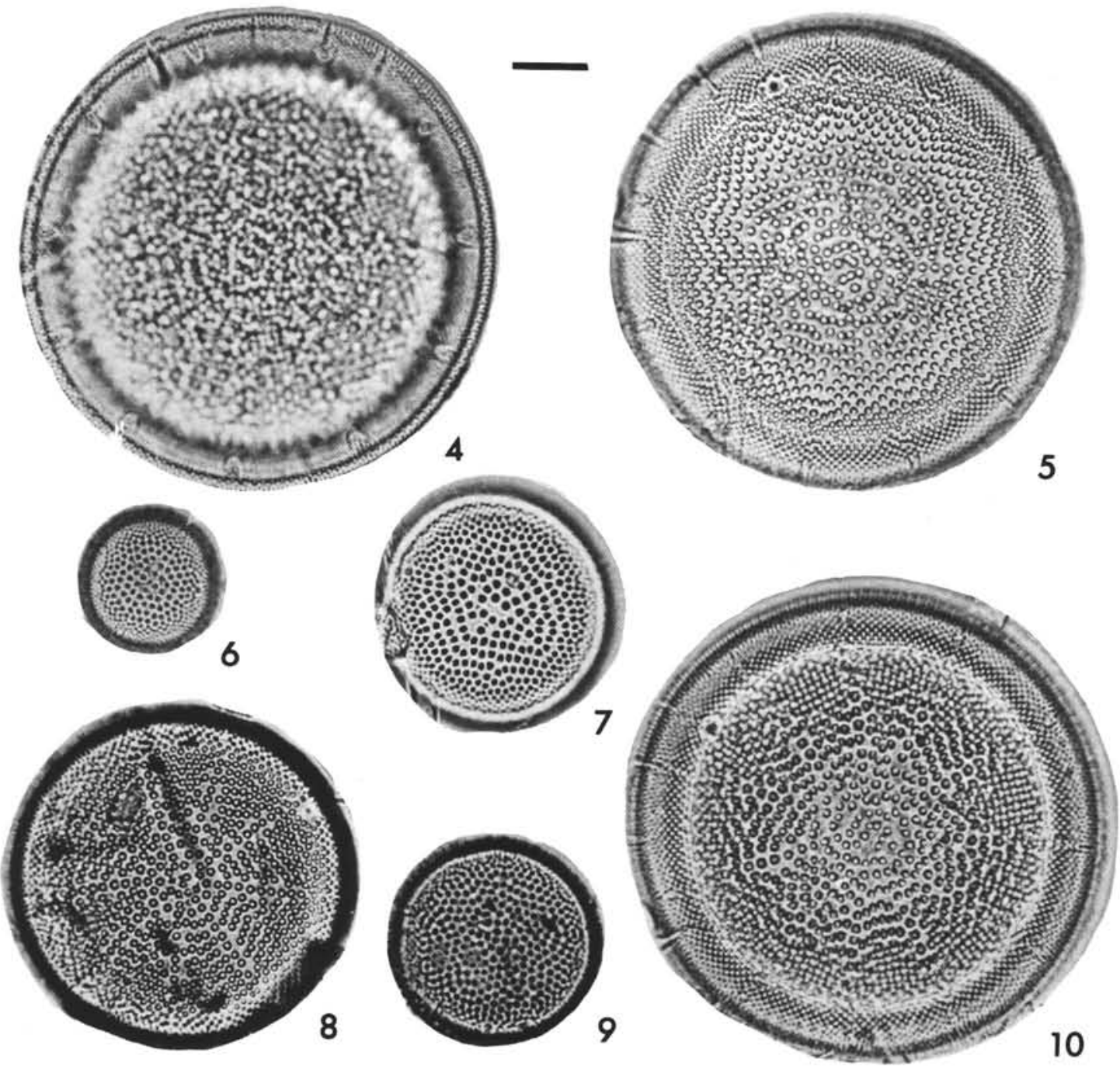


\section{PLATE 6}

Magnifications $\times 1000$

Scale bar represents 10 microns

Figure $1 \quad$ Rhososolenia sp. IV

Figure $2 \quad$ Bacteriastrum comosum Pavillard

Figures 3, $4 \quad$ Chaetoceros sp. VI.

Figure $5 \quad$ Chaetoceros gastridium Ehrenberg

Figure 6 Triceratium balearicum Cleve and Grunow var. biquadrata (Janisch) Hustedt

Figures 7,8 Grammatophora undulata Ehrenberg

Figures 9,11 Grammatophora oceanica (Ehrenberg) Grunow var. macilenta (W. Smith) Grunow

Figure 10 Grammatophora angulosa Ehrenberg var. islandica (Ehrenberg) Grunow

Figure 12 Grammatophora sp. I.

Figure $13 \quad$ Sceptroneis caducea Ehrenberg

Figures 14-16 fragilaria inflata (Heiden) Hustedt var. istvánffyi (Pantocsek) Hustedt

Figure $17 \quad$ Asterionella sp.

Figure $18 \quad$ Plagiogramma staurophorum (Gregory) Heiberg 
PLATE 6

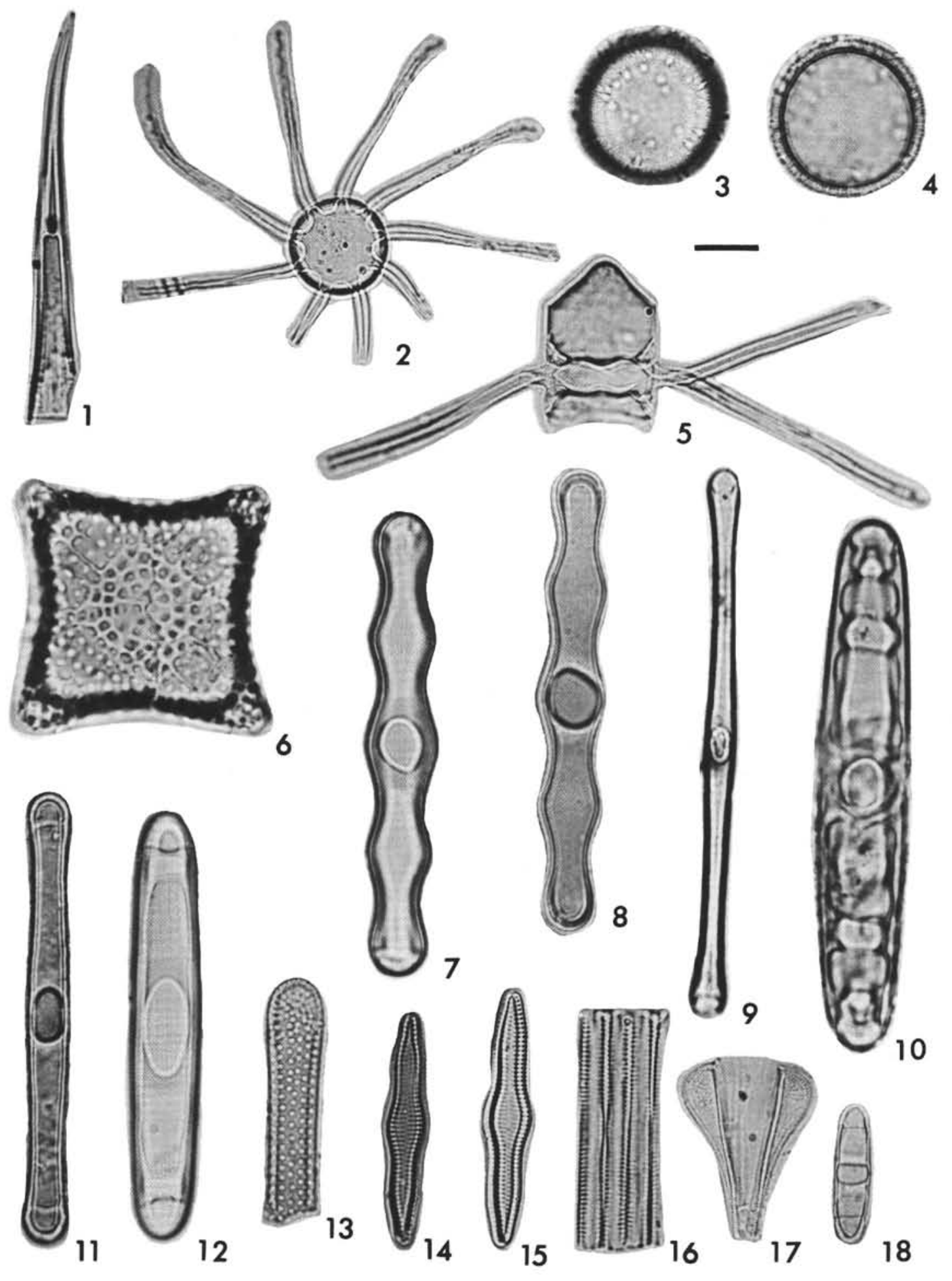


PLATE 7

Magnifications $\times 1000$

Scale bar represents 10 microns

Figure 1 Dimerogramma marinum (Gregory) Ralfs

Figure 2 Thalassionema nitzschioides Grunow

Figure 3 Fragilaria hirosakiensis Kanaya

Figure $4 \quad$ Campylosira sp.

Figure $5 \quad$ Cocconeis scutellum Ehrenberg

Figure $6 \quad$ Cocconeis sp. I

Figure $7 \quad$ Rhaphoneis surirella (Ehrenberg) Grunow

Figure $8 \quad$ Cocconeis britannica Naegeli

Figure 9 Cocconeis ornata Gregory var. signata Peragallo

Figure $10 \quad$ Cocconeis costata Gregory

Figure $11 \quad$ Cocconeis pediculus Ehrenberg

Figure $12 \quad$ Cocconeis scutellum Ehrenberg

Figure 13 Achnanthes fimbriata (Grunow) Ross

Figure $14 \quad$ Achnanthes brevipes Agardh

Figures 15-18 Mastogloia sp. II. 
PLATE 7
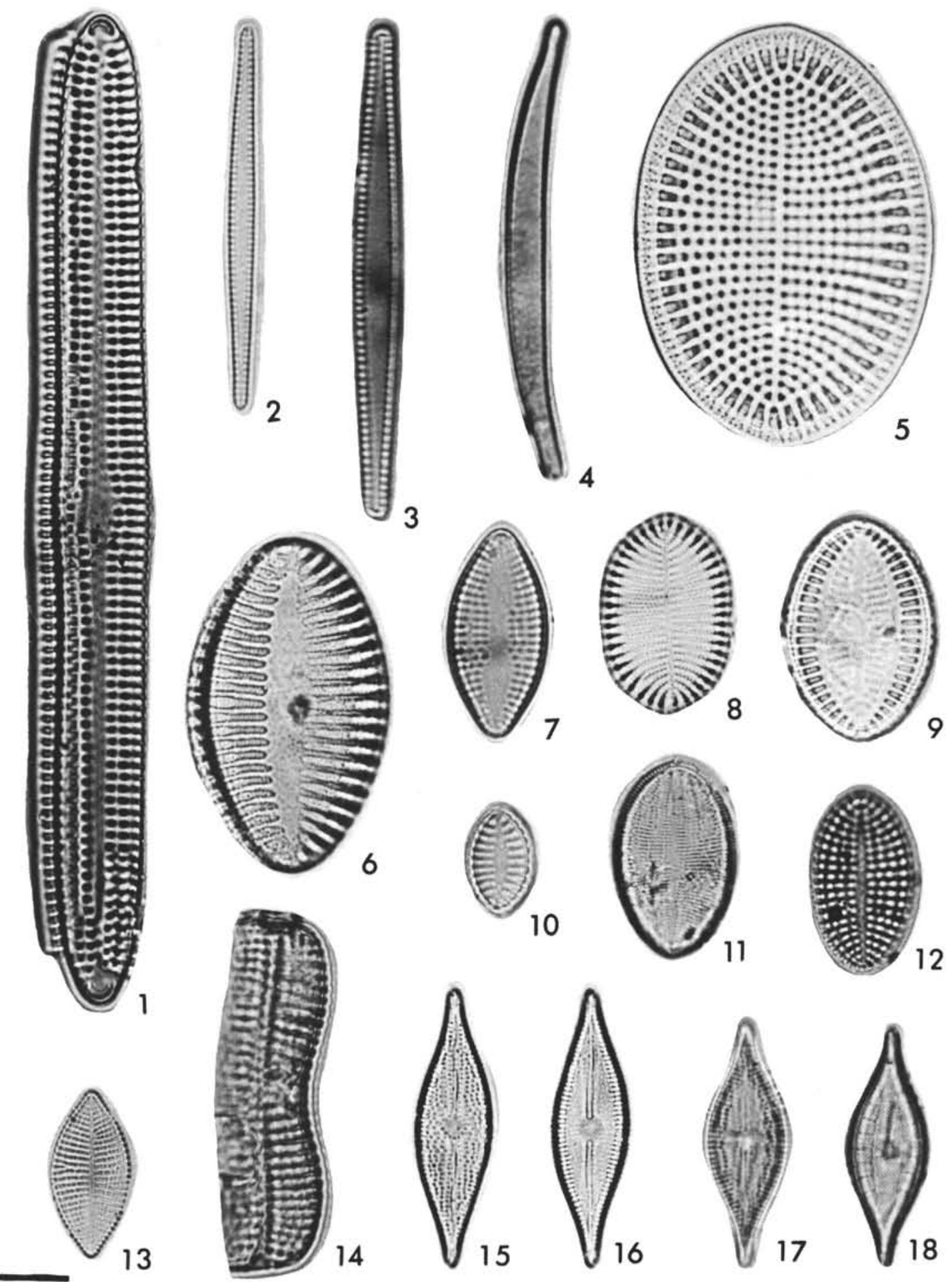
PLATE 8

Magnifications $\times 1000$

Scale bar represents 10 microns

Figures 1,2, 5 Mastogloia brauni Grunow

Figures 3, $4 \quad$ Mastogloia sp. II.

Figure 6 Diploneis gorjanovichi (Pantocsek) Hustedt

Figure 7 Diploneis ovalis (Hilse) Cleve

Figure $8 \quad$ Diploneis bombus Ehrenberg

Figure 9 Diploneis notabilis (Greville) Cleve

Figure $10 \quad$ Navicula comoides (Agardh) Peragallo

Figure $11 \quad$ Navicula pseudotuscula Hustedt

Figure $12 \quad$ Navicula impercepta Hustedt

Figures 13, 14 Denticula hustedtii Simonsen et Kanaya

Figure 15 Amphora minuta Pantocsek var. interrupta Hajós

Figure 16 Pinnularia eburnea (Carlson) Zanon

Figure 17 Amphora holsatica Hustedt

Figure 18 Navicula humerosa Brébisson 


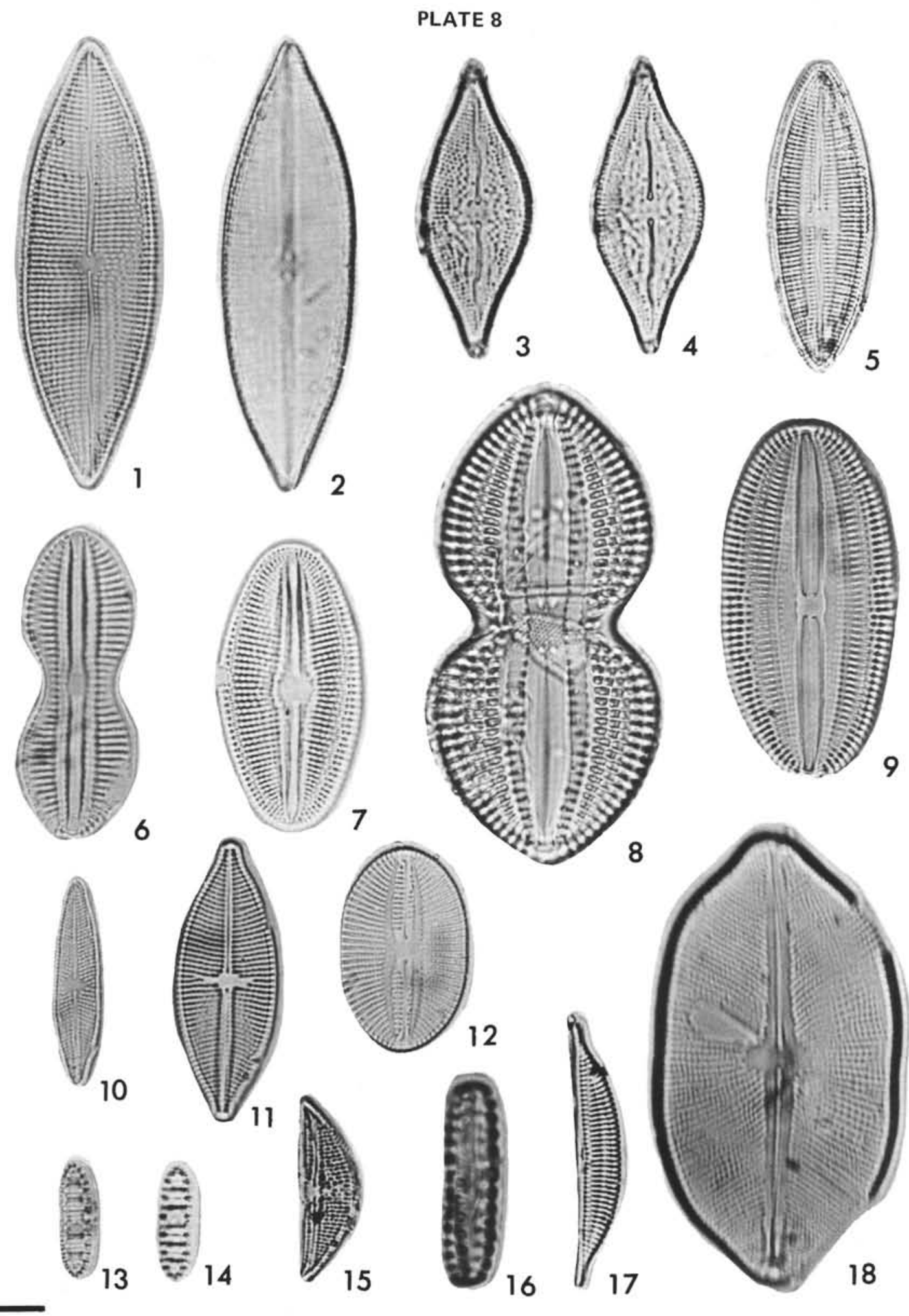


PLATE 9

Magnifications $\times 1000$

Scale bar represents 10 microns

Figure 1 Epithemia zebra (Ehrenberg) Kürtzing var. saxonica (Kürtzing) Grunow

Figure 2 Amphora sp.

Figure 3 Rhopalodia gibba (Ehrenberg) O. Müller. var. ventricosa (Ehrenberg) Grunow

Figure $4 \quad$ Epithemia sorex Kützing

Figure $5 \quad$ Epithemia turgida (Ehrenberg) Kützing

Figure $6 \quad$ Rhopalodia gibba (Ehrenberg) O. Müller

Figure 7,8 Porifera

Figure $9 \quad$ Pseudoeunotia doliolus (Wallich) Grunow

Figure $10 \quad$ Fragilariopsis pliocena (Brun) Sheshukova-Poretzskaya

Figure $11 \quad$ Nitzschia granulata Grunow

Figure 12 Campylodiscus clypeus Ehrenberg var. bicostata (W. Smith) Hustedt

Figure $13 \quad$ Cymatiosphaera cfr. miocaenica Hajós

Figures 14-17 Phytolitharia 
PLATE 9

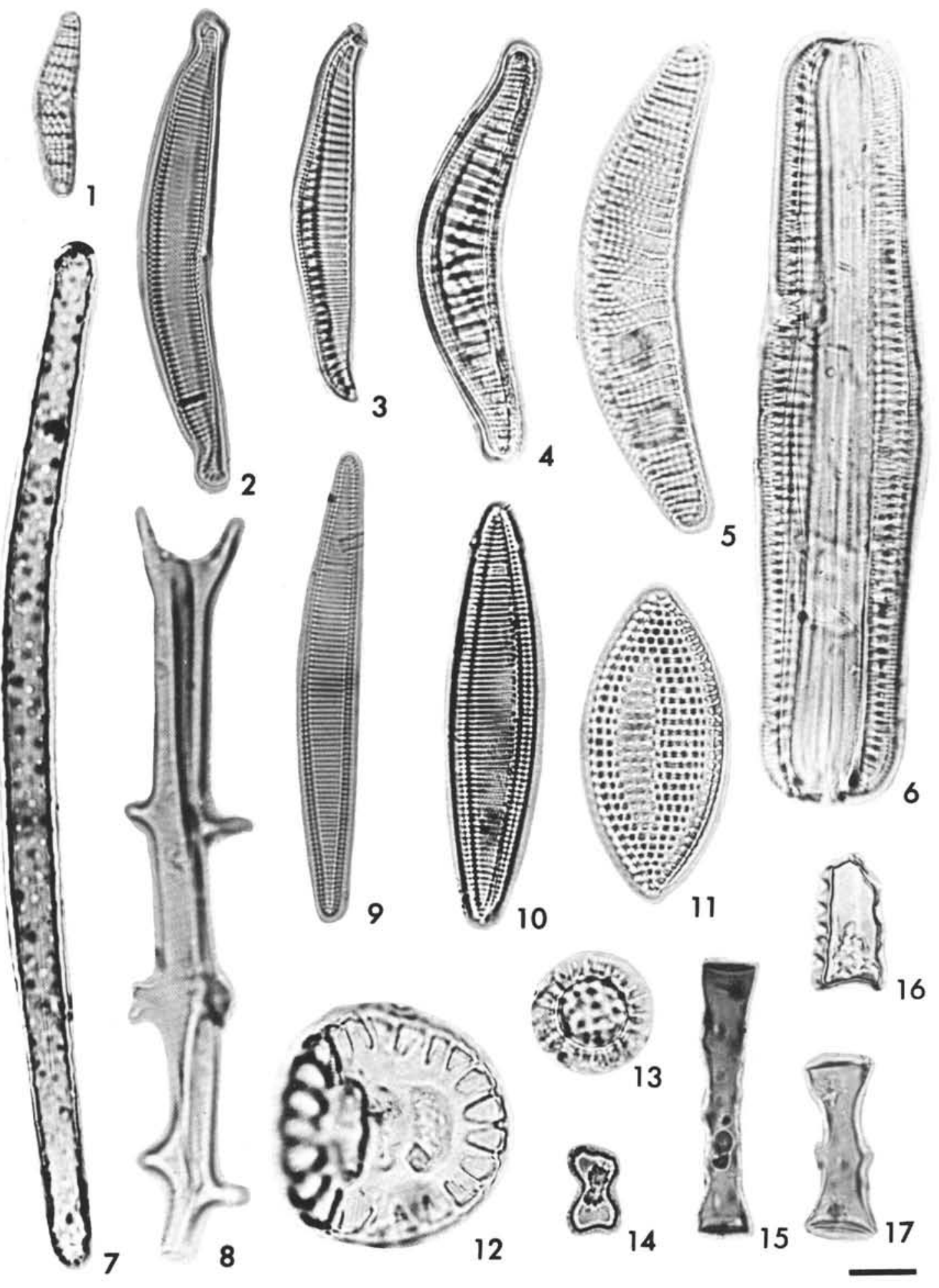

